\title{
A STUDY OF CONVEX CONVEX-COMPOSITE FUNCTIONS VIA INFIMAL CONVOLUTION WITH APPLICATIONS
}

\author{
JAMES V. BURKE, TIM HOHEISEL, AND QUANG V. NGUYEN
}

In memory of Jonathan M. Borwein

\begin{abstract}
In this note we provide a full conjugacy and subdifferential calculus for convex convex-composite functions in finite-dimensional space. Our approach, based on infimal convolution and cone-convexity, is straightforward and yields the desired results under a verifiable Slater-type condition, with relaxed monotonicity and without lower semicontinuity assumptions on the functions in play. The versatility of our findings is illustrated by a series of applications in optimization and matrix analysis, including conic programming, matrix-fractional, variational Gram, and spectral functions.
\end{abstract}

\section{Introduction}

Convex-composite optimization is a class of nonsmooth, nonconvex optimization problems that captures a wide variety of optimization models studied in modern optimization practice and theory including nonlinear programming (NLP), nonconvex minimax problems, nonconvex system identification, inverse problems and nonlinear filtering as well as most nonconvex problems in large-scale data analysis and machine learning, e.g. [27, 29, 30]. Given two Euclidean spaces $\mathbb{E}_{1}$ and $\mathbb{E}_{2}$, convex-composite problems take the form

$$
\min _{x \in \mathbb{E}_{1}} \Phi(x):=f(x)+g(F(x)),
$$

where $F: \mathbb{E}_{1} \rightarrow \mathbb{E}_{2}$ is continuous, usually smooth, while $f: \mathbb{E}_{1} \rightarrow \mathbb{R} \cup\{+\infty\}$ and $g: \mathbb{E}_{2} \rightarrow \mathbb{R} \cup\{+\infty\}$ are closed, proper, convex functions. The function $g$ encodes the modelling framework such as an NLP or an inverse problem and the function $f$ is a regularizer used to induce further properties on the solution such as sparsity, smoothness, or a domain restriction. The function $F$ is the functional data associated with a specific instance of the problem. In many treatments the function $f$ is often subsumed into the function $g$. This adds simplicity and is useful when establishing a number of theoretical results, a fact that we also exploit in our study.

The study of the convex-composite class began in the 1970's [45, 46] with major contributions occurring in the 1980's [10, 11, 37, 48, 49, 52, 53, and 1990's [14, 19, 25. 50. Recently there has been a resurgence of interest in the foundations of this problem class due to its importance for many problems in modern optimization, machine learning, and large scale data analysis [13, 17, 20, 23, 24, 26, 27, 29, 30, 42,

2010 Mathematics Subject Classification. 52A41, 65K10, 90C25, 90C46.

Key words and phrases. convex-composite function, cone-induced ordering, $K$-convexity, Fenchel conjugate, infimal convolution, subdifferential, conic programming, matrix-fractional function, variational Gram function, spectral function. 
In this note we study the case where $\Phi$ is convex. Although the convex-composite setting was proposed as a structure approach to nonconvex problems, understanding the convex case is important. This is illustrated in this paper by the applications in different areas of convex analysis and optimization which underline the relevance of our study.

So, when is $\Phi$ convex? We answer this with a simple result based on [12, Lemma 5.1]. The statement and proof are elementary, and so we give this result now since it motivates much of our subsequent investigation.

Theorem 1. Let the assumptions in (11) hold. Let $\alpha \in \mathbb{R}$ be such that $\operatorname{lev}_{g}(\alpha):=$ $\left\{y \in \mathbb{E}_{2} \mid g(y) \leq \alpha\right\} \neq \emptyset$ and define the horizon cone of $g$ by

$$
\text { hzn } g:=\left\{w \in \mathbb{E}_{2} \mid \forall t \geq 0: y+t w \in \operatorname{lev}_{g}(\alpha)\left(y \in \operatorname{lev}_{g}(\alpha)\right)\right\} \text {. }
$$

If $F$ is convex with respect to $-\mathrm{hzn} g$, i.e.

$$
F\left((1-\lambda) x_{1}+\lambda x_{2}\right)-\left[(1-\lambda) F\left(x_{1}\right)+\lambda F\left(x_{2}\right)\right] \in \operatorname{hzn} g \quad\left(x_{1}, x_{2} \in \mathbb{E}_{1}, \lambda \in[0,1]\right),
$$

then $\Phi$ is a convex function.

Proof. First recall that, by [47, Theorem 14.2], hzn $g$ is the polar cone of $\overline{c o n e}\left(\operatorname{dom} g^{*}\right)$, where $g^{*}$ is the convex conjugate of $g$, cf. (2) below. Hence, $F$ is convex with respect to - hzn $g$ if and only if

$$
\left\langle z, F\left((1-\lambda) x_{1}+\lambda x_{2}\right)\right\rangle \leq\left\langle z,(1-\lambda) F\left(x_{1}\right)+\lambda F\left(x_{2}\right)\right\rangle \quad\left(z \in \operatorname{dom} g^{*}\right),
$$

or equivalently,

$\left\langle z, F\left((1-\lambda) x_{1}+\lambda x_{2}\right)\right\rangle-g^{*}(z) \leq\left\langle z,(1-\lambda) F\left(x_{1}\right)+\lambda F\left(x_{2}\right)\right\rangle-g^{*}(z)\left(z \in \operatorname{dom} g^{*}\right)$.

Since $g=g^{* *}$ (as $g$ is closed, proper, convex) this proves the result.

This result illustrates the key features of our study: (i) the convexity of $F$ with respect to a cone $K$ and (ii) the convexity of $g$ and its monotonicity with respect to $K$. This structure is present in the most familiar result concerning the convexity of compositions which states that if $\mathbb{E}_{2}=\mathbb{R}$ and $g$ is nondecreasing and convex on its domain and $F$ is convex, then $\Phi$ is convex. In this case, clearly, $K=\mathbb{R}_{+}$. In particular, since $g$ is nondecreasing, hzn $g \subset \mathbb{R}_{-}$.

These two ideas, cone convexity and monotonicity, are certainly not new and are fundamental in convex analysis. Hence we now describe some of the most pertinent references for this study: Borwein [3] pursued an ambitious program of extending most of convex analysis to cone convex functions including conjugacy, subdifferential analysis, and duality, laying out much of the groundwork. Kusraev and Kutateladze [39] take this idea to an even more general setting by considering convex operators with values in arbitrary ordered vectors spaces. Combari, Laghdir, and Thibault [22] specifically study the convexity of $\Phi$ in the function space setting from the perspective of cone convexity, establishing conjugate and subdifferential formulas for $\Phi$ under Fenchel-Rockafellar and Attouch-Brézis-type qualification conditions, respectively. Pennanen [44] studies the convexity of $\Phi$ by developing a deep theory of generalized differentiation for graph-convex mappings, which yields some results on convex convex-composite functions as a by-product. More recently, based on an approach by Burachik et al. [9], Boţ, Grad, and Wanka [7. 8. provided a powerful characterization of the situation when the conjugate and subdifferential formulae established in [22, and in our study below, hold. 
Our approach to convex convex-composite functions differs from the above studies: With the exception of Pennanen [44, all the above references are set in infinite dimension. We work in finite-dimensional space which enables us to exploit the full strength of the relative topology of convex sets. This is particularly important with respect to our main convex-analytical workhorse, which is infimal convolution, see Section 2.1 for details. In contrast, the approach in the papers [22] and [7, 8, which are most closely related to our study, is via a pertubation function. Our approach via infimal convolution is more straightforward, hence more accessible. In addition, at least in the finite-dimensional setting, it yields more refined results with relaxed monotonicity and without lower semicontinuity of the functions in play. Our analysis is also facilitated in that we study the simple format $g \circ F$ first, and then extend it to the additive composite setting $f+g \circ F$. Thus, a main contribution of the paper is a simplified derivation of the central convex-analytical results for convex convex-composite functions, resulting in refined statements about conjugacy and subdifferentiation, under verifiable, point-based Slater-type conditions, see e.g. Theorem 12 and Corollary 14 for the simple $g \circ F$ setting, and Corollary 16 for the additive composite setting. Moreover, inspired by Theorem 1, we emphasize and study the case where $F$ is convex with resepect to the negative horizon cone of $g$, see Lemma 19 and Corollary 20. Given the significance of this case for the applications, see Section 5, this was certainly lacking in the above mentioned references on the topic. Another major contribution of our study is to illustrate the versatility and unifying character of the calculus of convex convex-composite setting by a series of applications of very different flavors. These go beyond what was considered before, and include connections to conic programming in Section 5.1, and to matrix analysis and modern matrix optimization, see Sections 5.35.5. More concretely, Section 5.3 contains a new extension of the matrix-fractional function [15, 16, 18] to the complex domain. Section 5.4 provides new, short proofs for the conjugate and subdifferential of variational Gram functions [16, 36, and Section 5.5] gives a new proof of Lewis' well-known result on spectral functions [40, 41] for the convex case. Section 5.6 extends a Farkas-type result due to Bot et al. 4].

Notation: Throughout $\mathbb{E}$ denotes a Euclidean space, i.e. a finite-dimensional, real vector space with inner product denoted by $\langle\cdot, \cdot\rangle$. For $A, B \subset \mathbb{E}$ their Minkowski sum is given by $A+B:=\{a+b \mid a \in A, b \in B\}$. In case $A=\{x\}$ we simply write $x+B:=\{x\}+B$. We write $\overline{\mathbb{R}}:=\mathbb{R} \cup\{ \pm \infty\}$ for the extended real line. For a complex number $z=a+b i(a, b \in \mathbb{R})$ and its complex conjugate $\bar{z}:=a-b i$, Re $z:=\frac{z+\bar{z}}{2}$ is its real part. Given a linear map $L: \mathbb{E}_{1} \rightarrow \mathbb{E}_{2}$, we write $L^{*}$ for its adjoint in the sense of linear algebra which is used accordingly for matrices. We denote the space of real and complex $n \times m$ matrices by $\mathbb{R}^{n \times m}$ and $\mathbb{C}^{n \times m}$, respectively. The $n \times n$ identity matrix is denoted by $I_{n}$. The space of Hermitian $n \times n$ matrices is denoted by $\mathbb{H}^{n}$ and the positive semidefinite and positive definite matrices in $\mathbb{H}^{n}$ are given by $\mathbb{H}_{+}^{n}$ and $\mathbb{H}_{++}^{n}$, respectively. We set $\mathbb{S}^{n}:=\mathbb{H}^{n} \cap \mathbb{R}^{n \times n}, \mathbb{S}_{+}^{n}:=\mathbb{H}_{+}^{n} \cap \mathbb{R}^{n \times n}$ and $\mathbb{S}_{++}^{n}:=\mathbb{H}_{++}^{n} \cap \mathbb{R}^{n \times n}$. The trace of $A \in \mathbb{C}^{n \times n}$ is denoted by $\operatorname{tr} A$.

\section{Preliminaries}

In what follows $\mathbb{E}$ denotes a Euclidean space, i.e. a finite-dimensional, real vector space with inner product denoted by $\langle\cdot, \cdot\rangle$. Given $S \subset \mathbb{E}$, its convex hull conv $S$ is the smallest convex set containing $S$. Given a convex set $C \subset \mathbb{E}$, its affine hull is the smallest affine set that contains $C$ or, equivalently, aff $C=\operatorname{span} C+\bar{x}$ for any 
$\bar{x} \in C$. The relative interior of $C$ is the interior in the topology relative to its affine hull, i.e. ri $C:=\left\{x \in C \mid \exists \varepsilon>0: B_{\varepsilon}(x) \cap\right.$ aff $\left.C \subset C\right\}$. The horizon (or recession) cone of (the convex set) $C$ is the closed, convex cone given by

$$
C^{\infty}:=\{v \in \mathbb{E} \mid \forall t>0: \bar{x}+t v \in \operatorname{cl} C(\bar{x} \in C)\} .
$$

We call a function $f: \mathbb{E} \rightarrow \overline{\mathbb{R}}$ convex if its epigraph epi $f:=\{(x, \alpha) \in \mathbb{E} \times \mathbb{R} \mid f(x) \leq \alpha\}$ is a convex set. We say that $f$ is proper if its domain $\operatorname{dom} f:=\{x \mid f(x) \leq+\infty\}$ is nonempty and $f$ does not take the value $-\infty$. We call $f$ lower semicontinuous (lsc) or closed if epi $f$ is closed. This is equivalent to saying that $f$ equals its closure or lower semicontinuous hull $\operatorname{cl} f: \mathbb{E} \rightarrow \overline{\mathbb{R}}$ given by

$$
(\operatorname{cl} f)(x):=\inf \left\{\alpha \mid \exists\left\{x_{k}\right\} \rightarrow x: f\left(x_{k}\right) \rightarrow \alpha\right\} \quad(x \in \mathbb{E}) .
$$

We employ the following abbreviations:

$$
\begin{aligned}
\Gamma(\mathbb{E}) & :=\{f: \mathbb{E} \rightarrow \mathbb{R} \cup\{+\infty\} \mid f \text { is proper and convex }\} \\
\Gamma_{0}(\mathbb{E}) & :=\{f \in \Gamma(\mathbb{E}) \mid f \text { is lower semicontinuous }\} .
\end{aligned}
$$

When the underlying space is clear, we simply write $\Gamma$ and $\Gamma_{0}$, respectively. A central operation that maps $\Gamma_{0}$ (one-to-one) to itself is Fenchel conjugation: For a function $f: \mathbb{E} \rightarrow \mathbb{R} \cup\{+\infty\}$ its (Fenchel) conjugate $f^{*}: \mathbb{E} \rightarrow \overline{\mathbb{R}}$ is given by

$$
f^{*}(y)=\sup _{x \in \mathbb{E}}\{\langle y, x\rangle-f(x)\} \quad(y \in \mathbb{E}) .
$$

The biconjugate of $f$ is $f^{* *}:=\left(f^{*}\right)^{*}$. It is well known, see e.g. [47, Theorem 12.2], that for $f \in \Gamma$ we have $\operatorname{cl} f=f^{* *} \in \Gamma_{0}$. The subdifferential of $f$ at $\bar{x} \in \operatorname{dom} f$ is the set

$$
\partial f(\bar{x}):=\{v \in \mathbb{E} \mid f(\bar{x})+\langle v, x-\bar{x}\rangle \leq f(x)(x \in \mathbb{E})\} .
$$

Subdifferential and conjugacy operator satisfy the Fenchel-Young inequality

$$
f(x)+f^{*}(v) \geq\langle x, v\rangle \quad(x, v \in \mathbb{E}),
$$

where

$$
f(x)+f^{*}(v)=\langle x, v\rangle \Longleftrightarrow v \in \partial f(x) \quad(x, v \in \mathbb{E}) .
$$

For $f \in \Gamma(\mathbb{E})$, and its horizon cone is given by hzn $f:=\operatorname{lev}_{f}(\alpha)^{\infty}$, where $\operatorname{lev}_{f}(\alpha):=$ $\{x \in \mathbb{E} \mid f(x) \leq \alpha\}$ is any nonempty (sub)level set of $f$ to the level $\alpha \in \mathbb{R}$.

2.1 Infimal convolution The most elementary, yet most central convexity-preserving functional operation is addition. It is paired in duality, in the sense of Fenchel conjugacy with infimal convolution, where for two functions $f, g: \mathbb{E} \rightarrow \mathbb{R} \cup\{+\infty\}$ their infimal convolution is defined by

$$
(f \square g)(x):=\inf _{y \in \mathbb{E}}\{f(y)+g(x-y)\} \quad(x \in \mathbb{E}) .
$$

If the infimum is attained for all $x \in \operatorname{dom} f \square g=\operatorname{dom} f+\operatorname{dom} g$, we slightly abuse notation and write

$$
(f \square g)(x)=\min _{y \in \mathbb{E}}\{f(y)+g(x-y)\} .
$$

The following classical result, see e.g. [47, Theorem 16.4], clarifies the conjugacy relation between addition and infimal convolution of convex functions. 
Theorem 2 (Conjugacy and infimal convolution). Let $f, g \in \Gamma(\mathbb{E})$. Then $(\operatorname{cl} f+$ $\operatorname{cl} g)^{*}=\operatorname{cl}\left(f^{*} \square g^{*}\right)$. If

$$
\operatorname{ri}(\operatorname{dom} f) \cap \operatorname{ri}(\operatorname{dom} g) \neq \emptyset
$$

then the closures are superfluous and the infimum is attained for all $y \in \operatorname{dom}\left(f^{*} \square g^{*}\right)$, i.e.

$$
(f+g)^{*}(y)=\min _{u \in \mathbb{E}}\left\{f^{*}(u)+g^{*}(y-u)\right\} .
$$

The second, most central convexity-preserving operation is (pre)-composition with a linear (affine) map: Given a closed, proper, convex function $g: \mathbb{E}_{2} \rightarrow \mathbb{R} \cup\{+\infty\}$ and a linear map $F: \mathbb{E}_{1} \rightarrow \mathbb{E}_{2}$, it is readily seen that the conjugate $(g \circ F)^{*}=\operatorname{cl}\left(F^{*} g^{*}\right)$ is the lower semicontinuous hull of the function

$$
\left(F^{*} g^{*}\right)(y)=\inf \left\{g^{*}(z) \mid F^{*}(z)=y\right\} .
$$

In order to ensure that the closure operation can be omitted, the relative interior condition

$$
F^{-1}(\operatorname{ri}(\operatorname{dom} g)) \neq \emptyset
$$

can be used, see [47, Theorem 16.3]. This result is, in essence, equivalent to Theorem 2. Combining the two, one arrives at the strong duality theorem of FenchelRockafellar duality for problems of the form

$$
\min _{x \in \mathbb{E}_{1}} f(x)+g(F(x)),
$$

Under the qualification condition

$$
0 \in \operatorname{ri}(\operatorname{dom} g-F(\operatorname{dom} f))
$$

it can be established that

$$
\inf _{x \in \mathbb{E}_{1}} f(x)+g(F(x))=\max _{y \in \mathbb{E}_{2}} f^{*}\left(F^{*}(y)\right)+g^{*}(-y) .
$$

Evidently, we are interested in the case of problem (5) where $F$ is not necessarily linear, but the composition $g \circ F$ is still convex, which is exactly the convex convexcomposite setting.

\section{$3 K$-convexity}

A subset $K \subset \mathbb{E}$ is called a cone if

$$
\lambda x \in K \quad(\lambda \geq 0, x \in K) .
$$

A cone $K$ is convex if and only if $K+K \subset K$ and it is (called) pointed if $K \cap(-K)=$ $\{0\}$. The polar (cone) of a cone $K$ is defined by $K^{\circ}:=\{v \in \mathbb{E} \mid\langle v, x\rangle \leq 0(x \in K)\}$. Given a cone $K \subset \mathbb{E}$ the relation

$$
x \leq_{K} y \quad: \Longleftrightarrow \quad y-x \in K \quad(x, y \in \mathbb{E})
$$

induces an ordering on $\mathbb{E}$ which is a partial ordering if $K$ is convex and pointed, see e.g. [50, Proposition 3.38]. We attach to $\mathbb{E}$ a largest element $+\infty$ • with respect to said ordering, which satisfies

$$
x \leq_{K}+\infty \cdot(x \in \mathbb{E}) .
$$


We will set $\mathbb{E}^{\bullet}:=\mathbb{E} \cup\{+\infty$ • $\}$. For a function $F: \mathbb{E}_{1} \rightarrow \mathbb{E}_{2}^{\bullet}$ its domain, graph and range are defined respectively as

$$
\begin{aligned}
\operatorname{dom} F & :=\left\{x \in \mathbb{E}_{1} \mid F(x) \in \mathbb{E}_{2}\right\}, \\
\operatorname{gph} F & :=\left\{(x, F(x)) \in \mathbb{E}_{1} \times \mathbb{E}_{2} \mid x \in \operatorname{dom} F\right\}, \\
\operatorname{rge} F & :=\left\{F(x) \in \mathbb{E}_{2} \mid x \in \operatorname{dom} F\right\} .
\end{aligned}
$$

We call $F$ proper if $\operatorname{dom} F \neq \emptyset$. The following concept is central to our study.

Definition 3 ( $K$-convexity). Let $K \subset \mathbb{E}_{2}$ be a cone and $F: \mathbb{E}_{1} \rightarrow \mathbb{E}_{2}^{\bullet}$. Then we call $F$ convex with respect to $K$ or $K$-convex if its $K$-epigraph

$$
K \text {-epi } F:=\left\{(x, v) \in \mathbb{E}_{1} \times \mathbb{E}_{2} \mid F(x) \leq_{K} v\right\}
$$

is convex (in $\mathbb{E}_{1} \times \mathbb{E}_{2}$ ).

We point out that, in the setting of Definition 3, a $K$-convex function $F$ has a convex domain as dom $F=L(K$-epi $F)$ where $L: \mathbb{E}_{1} \times \mathbb{E}_{2} \rightarrow \mathbb{E}_{1}:(x, v) \mapsto x$. Thus, $F$ is $K$-convex if and only if

$$
F(\lambda x+(1-\lambda) y) \leq_{K} \lambda F(x)+(1-\lambda) F(y) \quad(x, y \in \operatorname{dom} F, \lambda \in[0,1]) .
$$

Clearly, we can recover the traditional notion of a convex function $g: \mathbb{E} \rightarrow \mathbb{R} \cup\{+\infty\}$ by using $K=\mathbb{R}_{+}$in Definition 3 . Thus the following lemma is a generalization of the relative interior formula for the epigraph of an ordinary convex function, see e.g. [47, Lemma 7.3].

Lemma 4 (Relative interior of $K$-epigraph). Let $K \subset \mathbb{E}_{2}$ be a convex cone, and let $F: \mathbb{E}_{1} \rightarrow \mathbb{E}_{2}^{\bullet}$ be proper and $K$-convex. Then

$$
\begin{aligned}
\operatorname{ri}(K \text {-epi } F) & =\left\{(x, v) \mid x \in \operatorname{ri}(\operatorname{dom} F), F(x) \leq_{\text {ri }(K)} v\right\} \\
& =((\operatorname{ri} K) \text {-epi } F) \bigcap\left(\operatorname{ri}(\operatorname{dom} F) \times \mathbb{E}_{2}\right) .
\end{aligned}
$$

In particular, if $\operatorname{dom} F=\mathbb{E}_{1}$, then $\operatorname{ri}(K$-epi $F)=($ ri $K)$-epi $F$.

Proof. For $x \in \mathbb{E}_{1}$ set $C_{x}=\left\{v \in \mathbb{E}_{2} \mid(x, v) \in K\right.$-epi $\left.F\right\}$. It follows from [47, Theorem 6.7, Corollary 6.6.2] that

$$
\text { ri } C_{x}=\operatorname{ri}\left(L_{x}^{-1}(K)\right)=L_{x}^{-1}(\operatorname{ri} K)=\left\{v \in \mathbb{E}_{2} \mid v \in F(x)+\operatorname{ri} K\right\},
$$

where $L_{x}: v \mapsto v-F(x)$. Hence, by [47, Theorem 6.8], we find that

$$
\begin{aligned}
(x, v) \in \operatorname{ri}(K \text {-epi } F) & \Longleftrightarrow x \in \operatorname{ri}\left\{x \in \mathbb{E}_{1} \mid C_{x} \neq \emptyset\right\} \text { and } v \in \operatorname{ri} C_{x} \\
& \Longleftrightarrow x \in \operatorname{ri}(\operatorname{dom} F) \text { and } v \in \operatorname{ri} C_{x} \\
& \Longleftrightarrow x \in \operatorname{ri}(\operatorname{dom} F) \text { and } v \in F(x)+\operatorname{ri} K .
\end{aligned}
$$

We now turn our attention to the composite setting: For $F: \mathbb{E}_{1} \rightarrow \mathbb{E}_{2}^{\bullet}$ and $g: \mathbb{E}_{2} \rightarrow$ $\mathbb{R} \cup\{+\infty\}$ we define the composition of $g$ and $F$ as

$$
(g \circ F)(x)= \begin{cases}g(F(x)), & \text { if } x \in \operatorname{dom} F, \\ +\infty, & \text { else. }\end{cases}
$$

In particular, given $v \in \mathbb{E}_{2}$ and the linear form $\langle v, \cdot\rangle: \mathbb{E}_{2} \rightarrow \mathbb{R}$, we set $\langle v, F\rangle:=$ $\langle v, \cdot\rangle \circ F$, i.e.

$$
\langle v, F\rangle(x)= \begin{cases}\langle v, F(x)\rangle, & \text { if } x \in \operatorname{dom} F \\ +\infty, & \text { else. }\end{cases}
$$


This scalarization of $F$ is central to our study, a fact which is already foreshadowed in the next two results. These are in a similar form already present in the literature, e.g. 44, but we give the elementary proofs for completeness.

Theorem 5 (Scalarization of $K$-Convexity). Let $K \subset \mathbb{E}_{2}$ be a cone, let $F: \mathbb{E}_{1} \rightarrow \mathbb{E}_{2}^{\bullet}$ and $v \in \mathbb{E}_{2}$. Then the following hold:

a) If $F$ is $K$-convex then $\langle v, F\rangle$ is convex for all $v \in-K^{\circ}$.

b) The converse of a) holds true if $K$ is closed and convex.

Consequently, if $K$ is a closed, convex cone, then $F$ is $K$-convex if and only if $\langle v, F\rangle$ is convex for all $v \in-K^{\circ}$.

Proof. a) Suppose that $F$ is $K$-convex and let $v \in-K^{\circ}, x, y \in \operatorname{dom} F=\operatorname{dom}\langle v, F\rangle$, and $\lambda \in[0,1]$. Since $\lambda F(x)+(1-\lambda) F(y)-F(\lambda x+(1-\lambda) y) \in K$, it follows that

$$
\langle v, \lambda F(x)+(1-\lambda) F(y)-F(\lambda x+(1-\lambda) y)\rangle \geq 0
$$

and hence

$$
\begin{aligned}
\lambda\langle v, F\rangle(x)+(1-\lambda)\langle v, F\rangle(y) & =\langle v, \lambda F(x)+(1-\lambda) F(y)\rangle \\
& \geq\langle v, F(\lambda x+(1-\lambda) y)\rangle \\
& =\langle v, F\rangle(\lambda x+(1-\lambda) y) .
\end{aligned}
$$

b) Let $x, y \in \mathbb{E}_{1}, \lambda \in[0,1]$, and $v \in-K^{\circ}$. Since $\langle v, F\rangle$ is convex we have

$$
\begin{aligned}
0 & \leq \lambda\langle v, F\rangle(x)+(1-\lambda)\langle v, F\rangle(y)-\langle v, F\rangle(\lambda x+(1-\lambda) y) \\
& =\langle v, \lambda F(x)+(1-\lambda) F(y)-F(\lambda x+(1-\lambda) y)\rangle
\end{aligned}
$$

and therefore

$$
\lambda F(x)+(1-\lambda) F(y)-F(\lambda x+(1-\lambda) y) \in-\left(-K^{\circ}\right)^{\circ}=K
$$

as $K$ is closed and convex, see [47, Theorem 14.1].

For the next result recall that the indicator function of a nonempty set $S \subset \mathbb{E}$ is given by

$$
\delta_{S}: x \in \mathbb{E} \mapsto\left\{\begin{aligned}
0, & \text { if } \quad x \in S, \\
+\infty, & \text { else. }
\end{aligned}\right.
$$

Its conjugate is the support function $\sigma_{S}: \mathbb{E} \rightarrow \mathbb{R} \cup\{+\infty\}$ of $S$ given by

$$
\sigma_{S}(y)=\delta_{S}^{*}(y)=\sup _{x \in S}\langle x, y\rangle .
$$

Lemma 6. Let $F: \mathbb{E}_{1} \rightarrow \mathbb{E}_{2}^{\bullet}$ and let $K \subset \mathbb{E}_{2}$ be a closed, convex cone. Then the following hold for all $(u, v) \in \mathbb{E}_{1} \times \mathbb{E}_{2}$ :

a) $\sigma_{K \text {-epi } F}(u, v)=\sigma_{\operatorname{gph} F}(u, v)+\delta_{K \circ}(v)$.

b) $\sigma_{\operatorname{gph} F}(u,-v)=\langle v, F\rangle^{*}(u)$.

c) If $F$ is linear then $\langle v, F\rangle^{*}=\delta_{\left\{F^{*}(v)\right\}}$.

Proof. a) Observe that

$$
\begin{aligned}
\sigma_{K \text {-epi } F(u, v)}= & \sup _{(x, y) \in K \text {-epi } F}\langle(u, v),(x, y)\rangle \\
& =\sup _{(x, z) \in \mathbb{E}_{1} \times K}\langle(u, v),(x, F(x)+z)\rangle \\
& =\sup _{x \in \mathbb{E}_{1}}\langle(u, v),(x, F(x))\rangle+\sup _{z \in K}\langle z, v\rangle \\
& =\sigma_{\operatorname{gph} F}(u, v)+\delta_{K} \circ(v),
\end{aligned}
$$


where the last identity uses the well-known fact that $\sigma_{K}=\delta_{K}^{*}=\delta_{K}$ 。 for a closed, convex cone, see [50, Example 11.4].

b) We have

$$
\begin{aligned}
\sigma_{\mathrm{gph} F}(u,-v) & =\sup _{x \in \operatorname{dom} F}\langle(u,-v),(x, F(x))\rangle \\
& =\sup _{x \in \mathbb{E}_{1}}\{\langle u, x\rangle-\langle v, F\rangle(x)\} \\
& =\langle v, F\rangle^{*}(u) .
\end{aligned}
$$

c) By linearity of $F$ we have

$$
\langle v, F\rangle^{*}(u)=\sup _{x \in \mathbb{E}_{1}}\{\langle u, x\rangle-\langle v, F(x)\rangle\}=\sup _{x \in \mathbb{E}_{1}}\left\{\left\langle x, u-F^{*}(v)\right\rangle\right\}=\delta_{\left\{F^{*}(v)\right\}}(u) .
$$

The next result gives a sufficient condition for convexity of a composite function (6).

Proposition 7. Let $K \subset \mathbb{E}_{2}$ be a convex cone, $F: \mathbb{E}_{1} \rightarrow \mathbb{E}_{2}^{\bullet} K$-convex and $g \in \Gamma\left(\mathbb{E}_{2}\right)$. Then the following hold:

a) $g \circ F$ is proper if and only if $\operatorname{rge} F \cap \operatorname{dom} g \neq \emptyset$;

b) $g \circ F$ is convex if

$$
g(F(x)) \leq g(y) \quad((x, y) \in K \text {-epi } F) .
$$

Proof. a) Clear.

b) Let $v, w \in \operatorname{dom} F$ and $\lambda \in[0,1]$. Then

$$
\begin{aligned}
g(F(\lambda v+(1-\lambda) w)) & \leq g(\lambda F(v)+(1-\lambda) F(w)) \\
& \leq \lambda g(F(v))+(1-\lambda) g(F(w)) .
\end{aligned}
$$

Here the first inequality is due to the fact that $(\lambda v+(1-\lambda) w, \lambda F(v)+(1-\lambda) F(w)) \in$ $K$-epi $F$ (as $F$ is $K$-convex) and assumption (7).

We point out that the property (7) used to establish convexity of the composite function $g \circ F$, and which we borrowed from [44], is clearly weaker than saying that $g$ be $K$-increasing, i.e.

$$
g(u) \leq g(v) \quad\left(u \leq_{K} v\right),
$$

cf. Example 17 below. However, in Lemma 19 it will be shown that any closed, proper, convex function is increasing with respect to its own negative horizon cone. Condition (8) has some interesting consequences.

Lemma 8. Let $g: \mathbb{E}_{2} \rightarrow \mathbb{R} \cup\{+\infty\}$ satisfy (8) for some cone $K \subset \mathbb{E}_{2}$. Then $\operatorname{dom} g-K=\operatorname{dom} g$.

Proof. As $0 \in K$, we clearly have $\operatorname{dom} g-K \supset \operatorname{dom} g$. In turn, if $u \in \operatorname{dom} g$ and $v \in K$, by (8), we have $g(u-v) \leq g(u)$, which yields $u-v \in \operatorname{dom} g$.

We now investigate under which conditions we can also get closedness of the composite function. To this end, we first make an elementary observation.

Lemma 9. Let $K \subset \mathbb{E}$ be a cone and let $g: \mathbb{E} \rightarrow \overline{\mathbb{R}}$ be $K$-increasing in the sense of (8). Then $g=g \square \delta_{-K}$. 
Proof. For all $v \in \mathbb{E}$ we have

$$
\left(g \square \delta_{-K}\right)(v)=\inf _{u \in \mathbb{E}}\left\{g(v-u)+\delta_{-K}(u)\right\}=\inf _{u \in K} g(v+u)=g(v),
$$

where the last equality follows from (8).

The next result is a key observation which will be used multiple times in our study, but is also interesting in its own right.

Lemma 10. Let $K \subset \mathbb{E}_{2}$ be a closed, convex cone, let $F: \mathbb{E}_{1} \rightarrow \mathbb{E}_{2}^{\bullet}$ be such that $\left\{\langle v, F\rangle \mid v \in-K^{\circ}\right\} \subset \Gamma_{0}\left(\mathbb{E}_{1}\right)$, and let $g \in \Gamma_{0}\left(\mathbb{E}_{2}\right)$ be $K$-increasing. Define $\psi$ : $\mathbb{E}_{1} \times \mathbb{E}_{2} \rightarrow \mathbb{R} \cup\{+\infty\}$,

$$
\psi(p, v):=g^{*}(v)+\sigma_{K \text {-epi } F}(p,-v) .
$$

Then $\psi^{*}(x, u)=g(u+F(x))$.

Proof. We have

$$
\begin{aligned}
\psi^{*}(x, u) & =\sup _{(p, v) \in \mathbb{E}_{1} \times \mathbb{E}_{2}}\{\langle x, p\rangle+\langle u, v\rangle-\psi(p, v)\} \\
& =\sup _{(p, v) \in \mathbb{E}_{1} \times \mathbb{E}_{2}}\left\{\langle x, p\rangle+\langle u, v\rangle-g^{*}(v)-\langle v, F\rangle^{*}(p)-\delta_{-K^{\circ}}(v)\right\} \\
& =\sup _{v \in \mathbb{E}_{2}}\left\{\langle u, v\rangle-g^{*}(v)-\delta_{-K^{\circ}}(v)+\sup _{p \in \mathbb{E}_{1}}\left\{\langle x, p\rangle-\langle v, F\rangle^{*}(p)\right\}\right\} \\
& =\sup _{v \in \mathbb{E}_{2}}\left\{\langle u, v\rangle-g^{*}(v)-\delta_{-K^{\circ}}(v)+\langle v, F\rangle(x)\right\} \\
& =\sup _{v \in \mathbb{E}_{2}}\left\{\langle u+F(x), v\rangle-g^{*}(v)-\delta_{-K^{\circ}}(v)\right\} \\
& =\left(g^{*}+\delta_{-K^{\circ}}\right)^{*}(u+F(x)) \\
& =\left(g \square \delta_{-K}\right)^{* *}(u+F(x)) \\
& =g(u+F(x)) .
\end{aligned}
$$

Here the second identity comes from Lemma 6 a), the fourth uses the fact that $\langle v, F\rangle^{* *}=\langle v, F\rangle\left(v \in-K^{\circ}\right)$ by assumption. The last before last identity employs Theorem 2 and the fact that $\left(g \square \delta_{-K}\right)^{* *}=\operatorname{cl}\left(g \square \delta_{-K}\right)$. The last equality follows from Lemma 9 and using $g^{* *}=g$.

We close out this section with sufficient conditions for closedness (and convexity) of the composite functions from ([6).

Corollary 11. Under the assumptions of Lemma 10 the composite function $g \circ F$ is closed and convex. In particular, if, in addition, $\operatorname{rge} F \cap \operatorname{dom} g \neq \emptyset$, then $g \circ F \in$ $\Gamma_{0}\left(\mathbb{E}_{1}\right)$.

Proof. By Lemma 10 we have $g \circ F=\psi^{*}(\cdot, 0)$, which is closed and convex. The properness is exactly the additional assumption.

We point out that Corollary 11 generalizes the well-established scalar case as in e.g. [33. Proposition 2.1.7, Chapter B]. 


\section{Convex analysis of convex convex-composite functions}

We commence with the main result of this section. Throughout, we use the standing assumption that the function $F: \mathbb{E}_{1} \rightarrow \mathbb{E}_{2}^{\bullet}$ in question is proper, i.e. $\operatorname{dom} F \neq \emptyset$.

Theorem 12 (Conjugate of composite function). Let $K \subset \mathbb{E}_{2}$ be a closed, convex cone, $F: \mathbb{E}_{1} \rightarrow \mathbb{E}_{2}^{\bullet} K$-convex and $g \in \Gamma\left(\mathbb{E}_{2}\right)$ such that (7) is satisfied. Then the following hold:

a) $(g \circ F)^{*} \leq \operatorname{cl} \eta$, where

$$
\eta(p)=\inf _{v \in-K^{\circ}} g^{*}(v)+\langle v, F\rangle^{*}(p)
$$

Equality holds if, in addition, $\langle v, F\rangle \in \Gamma_{0}\left(\mathbb{E}_{2}\right)$ for all $v \in-K^{\circ}, g$ is lower semicontinuous and $K$-increasing, and $\operatorname{rge} F \cap \operatorname{dom} g \neq \emptyset$.

b) If

$$
F(\operatorname{ri}(\operatorname{dom} F)) \cap \operatorname{ri}(\operatorname{dom} g-K) \neq \emptyset .
$$

the function $\eta$ in a) is closed, proper and convex and the infimum is a minimum (possibly $+\infty$ ).

c) Under the assumptions of b) we have

$$
(g \circ F)^{*}(p)=\min _{v \in-K^{\circ}} g^{*}(v)+\langle v, F\rangle^{*}(p)
$$

with $\operatorname{dom}(g \circ F)^{*}=\left\{p \in \mathbb{E}_{1} \mid \exists v \in \operatorname{dom} g^{*} \cap\left(-K^{\circ}\right):\langle v, F\rangle^{*}(p)<+\infty\right\}$.

Proof. a) Define $\phi: \mathbb{E}_{1} \times \mathbb{E}_{2} \rightarrow \mathbb{R} \cup\{+\infty\}:(x, y) \mapsto g(y)$ and observe that

$$
\phi^{*}(u, v)=\delta_{\{0\}}(u)+g^{*}(v) .
$$

Hence we find that

$$
\begin{aligned}
& (g \circ F)^{*}(p)=\sup _{x \in \mathbb{E}_{1}}\{\langle x, p\rangle-g(F(x))\} \\
& =\sup _{(x, y) \in K \text {-epi } F}\{\langle x, p\rangle-g(y)\} \\
& =\sup _{(x, y) \in \mathbb{E}_{1} \times \mathbb{E}_{2}}\left\{\langle(p, 0),(x, y)\rangle-\left(g(y)+\delta_{K \text {-epi } F}(x, y)\right\}\right. \\
& =\left(\phi+\delta_{K \text {-epi } F}\right)^{*}(p, 0) \\
& \leq\left(\operatorname{cl} \phi+\operatorname{cl} \delta_{K \text {-epi } F)^{*}(p, 0)}\right. \\
& =\operatorname{cl}\left(\phi^{*} \square \sigma_{K \text {-epi } F}\right)(p, 0) \text {. }
\end{aligned}
$$

Here the second equality uses assumption (77), and the inequality is due to the fact that conjugation is order-reversing. The last identity is then due to Theorem 2 as the functions in play are proper and convex by assumption. Moreover, we have

$$
\begin{aligned}
\left(\phi^{*} \square \sigma_{K \text {-epi } F}\right)(p, 0) & =\inf _{(u, v) \in \mathbb{E}_{1} \times \mathbb{E}_{2}}\left\{\phi^{*}(u, v)+\sigma_{K \text {-epi } F}(p-u,-v)\right\} \\
& =\inf _{v \in \mathbb{E}_{2}}\left\{g^{*}(v)+\sigma_{K \text {-epi } F}(p,-v)\right\} \\
& =\inf _{v \in-K^{\circ}}\left\{g^{*}(v)+\sigma_{\operatorname{gph} F}(p,-v)\right\} \\
& =\inf _{v \in-K^{\circ}}\left\{g^{*}(v)+\langle v, F\rangle^{*}(p)\right\},
\end{aligned}
$$

where the second identity uses (11) and the third and fourth rely on Lemma 6] This shows the first desired statement.

To show that equality holds under the additional assumptions observe that with the function $\psi$ in (9), we have $\eta=\inf _{v} \psi(\cdot, v)$. Hence $\eta$ is convex and, by Lemma10. 
it holds that $\eta^{*}=\psi^{*}(\cdot, 0)=g \circ F$. As $g \circ F$ is closed, proper, convex by Corollary 11 $\eta$ is convex and proper, and so $\operatorname{cl} \eta=\eta^{* *}=(g \circ F)^{*}$, which is the desired equality.

b) This follows from Theorem 2 while observing that

$$
\begin{aligned}
\operatorname{ri}(\operatorname{dom} \phi) \cap \operatorname{ri}\left(\operatorname{dom} \delta_{K \text {-epi } F) \neq \emptyset}\right. & \Longleftrightarrow \mathbb{E}_{1} \times \operatorname{ri}(\operatorname{dom} g) \cap \operatorname{ri}(K \text {-epi } F) \neq \emptyset \\
& \Longleftrightarrow \exists x \in \operatorname{ri}(\operatorname{dom} F): F(x) \in \operatorname{ri}(\operatorname{dom} g)-\operatorname{ri} K \\
& \Longleftrightarrow F(\operatorname{ri}(\operatorname{dom} F)) \cap \operatorname{ri}(\operatorname{dom} g-K) \neq \emptyset .
\end{aligned}
$$

Here the second equivalence relies on Lemma 4.

c) The first statement follows from a) and b) and Theorem 2. The expression of $\operatorname{dom}(g \circ F)^{*}$ is an immediate consequence of that.

We would like to point out that the technique of proof based on infimal convolution is an extension of the approach employed by Hiriart-Urruty in 32. However, our setting is much more general, and our point-based, Slater-type qualification condition (10) differs substantially from the Fenchel-Rockafellar-type condition used in 32 .

We now continue with a whole sequence of rather immediate consequences of Theorem 12. The first one is a subdifferential formula. To this end, we first establish an auxiliary result.

Lemma 13. Let $K \subset \mathbb{E}_{2}$ be a closed, convex cone. Then the following hold:

a) Let $g: \mathbb{E}_{2} \rightarrow \mathbb{R} \cup\{+\infty\}$ such that (8) holds and let $\bar{x} \in F^{-1}(\operatorname{dom} g)$. Then $\partial g(F(\bar{x})) \subset-K^{\circ}$.

b) Under the assumptions of Theorem 12 c) let $\bar{y} \in \partial(g \circ F)(\bar{x})$ and $\bar{v} \in$ $\operatorname{argmin}_{v \in-K^{\circ}}\left\{g^{*}(v)+\langle v, F\rangle^{*}(\bar{y})\right\}$. Then $\bar{v} \in \partial g(F(\bar{x}))$.

Proof. a) Let $\bar{v} \in \partial g(F(\bar{x}))$, i.e.

$$
g(F(\bar{x}))+\langle\bar{v}, y-F(\bar{x})\rangle \leq g(y) \quad\left(y \in \mathbb{E}_{2}\right) .
$$

In particular, letting $y=F(\bar{x})-k(k \in K)$ and by (8), this implies

$$
0 \leq g(F(\bar{x}))-g(F(\bar{x})-k) \leq\langle\bar{v}, k\rangle \quad(k \in K) .
$$

Therefore, $\bar{v} \in-K^{\circ}$.

b) We observe that

$$
\begin{aligned}
\langle\bar{x}, \bar{y}\rangle & =(g \circ F)(\bar{x})+(g \circ F)^{*}(\bar{y}) \\
& =g(F(\bar{x}))+g^{*}(\bar{v})+\sigma_{\operatorname{gph} F}(\bar{y},-\bar{v}) \\
& =g(F(\bar{x}))+g^{*}(\bar{v})+\sup _{z \in \mathbb{E}_{1}}\langle z, \bar{y}\rangle-\langle F(z), \bar{v}\rangle .
\end{aligned}
$$

Here the first identity is due to (4), and the second is due to Theorem 12 c) in combination with Lemma 6, Now insert $z:=\bar{x}$ to obtain

$$
\begin{aligned}
& \langle\bar{x}, \bar{y}\rangle \geq g(F(\bar{x}))+g^{*}(\bar{v})+\langle\bar{x}, \bar{y}\rangle-\langle F(\bar{x}), \bar{v}\rangle \\
\Longleftrightarrow & \langle F(\bar{x}), \bar{v}\rangle \geq g(F(\bar{x}))+g^{*}(\bar{v}) \\
\Longleftrightarrow & \bar{v} \in \partial g(F(\bar{x})) .
\end{aligned}
$$


Corollary 14 (Subdifferential of composite functions). For $g: \mathbb{E}_{2} \rightarrow \mathbb{R} \cup\{+\infty\}$ and $F: \mathbb{E}_{1} \rightarrow \mathbb{E}_{2}^{\bullet}$ we have

$$
\partial(g \circ F)(\bar{x}) \supset \bigcup_{v \in \partial g(F(\bar{x}))} \partial\langle v, F\rangle(\bar{x}) \quad(\bar{x} \in \operatorname{dom} g \circ F) .
$$

Equality holds under the assumptions of Theorem $12 \mathrm{~b}$ ).

Proof. Let $\bar{y} \in \bigcup_{v \in \partial g(F(\bar{x}))} \partial\langle v, F\rangle(\bar{x})$, i.e. there exists $\bar{v} \in \partial g(F(\bar{x}))$ such that $\bar{y} \in \partial\langle\bar{v}, F\rangle(\bar{x})$. Therefore

$$
g(F(\bar{x}))+\langle\bar{v}, y-F(\bar{x})\rangle \leq g(y) \quad\left(y \in \mathbb{E}_{2}\right)
$$

and

$$
\langle\bar{v}, F(\bar{x})\rangle+\langle\bar{y}, x-\bar{x}\rangle \leq\langle\bar{v}, F(x)\rangle \quad\left(x \in \mathbb{E}_{1}\right) .
$$

Inserting $y:=F(x)\left(x \in \mathbb{E}_{1}\right)$ in (14) yields

$$
g(F(\bar{x}))+\langle\bar{v}, F(x)-F(\bar{x})\rangle \leq g(F(x)) \quad\left(x \in \mathbb{E}_{1}\right) .
$$

Combining with (15) now gives

$$
g(F(\bar{x}))+\langle\bar{y}, x-\bar{x}\rangle \leq g(F(x)) \quad\left(x \in \mathbb{E}_{1}\right),
$$

which shows $\bar{y} \in \partial(g \circ F)(\bar{x})$.

To see the reverse inclusion under the additional assumptions let $\bar{y} \in \partial(g \circ F)(\bar{x})$, i.e., by (44), we have

$$
\langle\bar{x}, \bar{y}\rangle=(g \circ F)(\bar{x})+(g \circ F)^{*}(\bar{y}) .
$$

Now let $\bar{v} \in \operatorname{argmin}_{v \in-K^{\circ}}\left\{g^{*}(v)+\langle v, F\rangle^{*}(\bar{y})\right\}$. Then from (16) we infer

$$
\begin{aligned}
\langle\bar{x}, \bar{y}\rangle & =g(F(\bar{x}))+g^{*}(\bar{v})+\langle\bar{v}, F\rangle^{*}(\bar{y}) \\
& =\langle F(\bar{x}), \bar{v}\rangle+\langle\bar{v}, F\rangle^{*}(\bar{y}) \\
& =\langle\bar{v}, F\rangle(\bar{x})+\langle\bar{v}, F\rangle^{*}(\bar{y}),
\end{aligned}
$$

where the first identity is due to the choice of $\bar{v}$ and the second is (4) with Lemma $13 \mathrm{~b})$. This yields $\bar{y} \in \partial\langle\bar{v}, F\rangle(\bar{x})$.

Remark 15. Given $F: \mathbb{E}_{1} \rightarrow \mathbb{E}_{2} K$-convex and $v \in-K^{\circ}$, the convex function $\langle v, F\rangle$ enjoys a rich (sub)differential calculus under additional assumptions on the continuity or differentiability of $F$. For instance, if $F$ is locally Lipschitz, then so is $\langle v, F\rangle$ with

$$
\partial\langle v, F\rangle(\bar{x})=\bar{\partial} F(\bar{x})^{*} v,
$$

where $\bar{\partial} F(\bar{x})$ is Clarke's generalized Jacobian of $F$ at $\bar{x}$, see [21, 50]. In particular, if $F$ is differentiable, then $\langle v, F\rangle$ is continuously differentiable with

$$
\nabla\langle v, F\rangle(\bar{x})=\nabla F(\bar{x})^{*} v .
$$

The next result shows that our setting $g \circ F$ in fact covers the seemingly more general setting from (11) with an additional additive term. Once more, the proof relies essentially on infimal convolution.

Corollary 16 (Conjugate and subdifferential of additive composite functions). Under the assumptions of Theorem 12 let (7) hold. In addition, let $f \in \Gamma$ and consider the qualification condition

$$
F(\operatorname{ri}(\operatorname{dom} f) \cap \operatorname{ri}(\operatorname{dom} F)) \cap \operatorname{ri}(\operatorname{dom} g-K) \neq \emptyset .
$$

Then the following hold: 
a) If (17) holds then

$$
(f+g \circ F)^{*}(p)=\min _{\substack{v \in-K^{\circ}, y \in \mathbb{E}_{1}}} g^{*}(v)+f^{*}(y)+\langle v, F\rangle^{*}(p-y),
$$

b) We have

$$
\partial(f+g \circ F)(\bar{x}) \supset \partial f(\bar{x})+\bigcup_{v \in \partial g(F(\bar{x}))} \partial\langle v, F\rangle(\bar{x}) \quad(\bar{x} \in \operatorname{dom} f \cap \operatorname{dom} g \circ F) .
$$

Equality holds under (17).

Proof. a) Define $\tilde{g}: \mathbb{R} \times \mathbb{E}_{2} \rightarrow \mathbb{R} \cup\{+\infty\}$ by $\tilde{g}(s, y):=s+g(y)$. Then $\tilde{g} \in \Gamma_{0}\left(\mathbb{R} \times \mathbb{E}_{2}\right)$ with

$$
\tilde{g}^{*}(t, v)=\delta_{\{1\}}(t)+g^{*}(v) .
$$

Moreover, define $\tilde{F}: \mathbb{E}_{1} \rightarrow \mathbb{R} \cup\{+\infty\} \times \mathbb{E}_{2}^{\bullet}$ by $\tilde{F}(x)=(f(x), F(x))$. Then $\operatorname{dom} \tilde{F}=$ $\operatorname{dom} f \cap \operatorname{dom} F$. Setting $\tilde{K}:=\mathbb{R}_{+} \times K$, we find that $\tilde{F}$ is $\tilde{K}$-convex, $\tilde{g} \circ \tilde{F}=$ $f+g \circ F \in \Gamma_{0}\left(\mathbb{E}_{1}\right)$ and $\tilde{g} \circ \tilde{F}$ satisfies (7) with $\tilde{K}$ (since $g \circ F$ satisfies it with $K$ ). Moreover, as $\operatorname{dom} \tilde{g}=\mathbb{R} \times \operatorname{dom} g$, we realize that condition (10) for $\tilde{g}, \tilde{F}$ and $\tilde{K}$ amounts to (17). Therefore, we obtain

$$
\begin{aligned}
(f+g \circ F)^{*}(p) & =(\tilde{g} \circ \tilde{F})(p) \\
& =\min _{(t, v) \in-\tilde{K}^{\circ}} \tilde{g}^{*}(t, v)+\langle(t, v), \tilde{F}\rangle^{*}(p) \\
& =\min _{v \in-K^{\circ}} g^{*}(v)+\sup _{x \in \mathbb{E}_{1}}\{\langle p, v\rangle-(f(x)+\langle v, F\rangle(x))\} \\
& =\min _{v \in-K^{\circ}} g^{*}(v)+(f+\langle v, F\rangle)^{*}(p) \\
& =\min _{v \in-K^{\circ}} g^{*}(v)+\left(f^{*} \square\langle v, F\rangle^{*}\right)(p) \\
& =\min _{\substack{v \in-K^{\circ} \\
y \in \mathbb{E}_{1}}} g^{*}(v)+f^{*}(y)+\langle v, F\rangle^{*}(p-y) .
\end{aligned}
$$

Here the second identity is due to Theorem 12, the third one uses (18), while the fifth relies once more on Theorem 2, realizing that $\operatorname{dom}\langle v, F\rangle=\operatorname{dom} F$ and (17) implies that $\operatorname{ri}(\operatorname{dom} f) \cap \operatorname{ri}(\operatorname{dom} F) \neq \emptyset$.

b) The first statement (without qualification condition) follows from Corollary 14 and the subdifferential sum rule [47, Theorem 23.8].

In order to prove the converse inclusion under (17), let $\bar{y} \in \partial(f+g \circ F)(\bar{x})$. Hence by (4) and (19) we have

$$
\langle\bar{x}, \bar{y}\rangle-\left(f(x)+g(F(\bar{x}))=(f+g \circ F)^{*}(\bar{y})=\min _{v \in-K^{\circ}} g^{*}(v)+(f+\langle v, F\rangle)^{*}(\bar{y}) .\right.
$$

Hence there exists $\bar{v} \in-K^{\circ}$ such that

$$
(f+g \circ F)^{*}(\bar{y})=g^{*}(\bar{v})+(f+\langle\bar{v}, F\rangle)^{*}(\bar{y}) .
$$

Therefore, we have

$$
\langle\bar{x}, \bar{y}\rangle=f(\bar{x})+g(F(\bar{x}))+g^{*}(\bar{v})+(f+\langle\bar{v}, F\rangle)^{*}(\bar{y}) .
$$

On the one hand, (21) and the Fenchel-Young inequality (3) applied to $g$ imply

$$
\langle\bar{x}, \bar{y}\rangle \geq f(\bar{x})+\langle\bar{v}, F(\bar{x})\rangle+(f+\langle\bar{v}, F\rangle)^{*}(\bar{y}),
$$


which, by (4) applied to $f+\langle\bar{v}, F\rangle$, yields $\bar{y} \in \partial(f+\langle\bar{v}, F\rangle)(\bar{x})$. On the other hand, we deduce from (21) and the definition of the conjugate that

$$
\langle\bar{x}, \bar{y}\rangle \geq f(\bar{x})+g(F(\bar{x}))+g^{*}(v)+\langle\bar{x}, \bar{y}\rangle-f(\bar{x})-\langle\bar{v}, F(\bar{x})\rangle,
$$

which, in turn, yields

$$
\langle\bar{v}, F(\bar{x})\rangle \geq g(F(\bar{x}))+g^{*}(\bar{v}),
$$

and hence, $\bar{v} \in \partial g(F(\bar{x}))$, by (4). Altogether, we deduce that

$$
\begin{aligned}
\partial(f+g \circ F)(\bar{x}) & \subset \bigcup_{v \in \partial g(F(\bar{x}))} \partial(f+\langle v, F\rangle)(\bar{x}) \\
& =\bigcup_{v \in \partial g(F(\bar{x}))}(\partial f(\bar{x})+\partial\langle v, F\rangle(\bar{x})) \\
& =\partial f(\bar{x})+\bigcup_{v \in \partial g(F(\bar{x}))} \partial\langle v, F\rangle(\bar{x}) \\
& \subset \partial f(\bar{x})+\partial(g \circ F)(\bar{x}),
\end{aligned}
$$

and hence, the conclusion follows. Here, the first equality follows from the subdifferential sum rule, see e.g. [47, Theorem 23.8] because $\operatorname{ri}(\operatorname{dom} f) \cap \operatorname{ri}(\operatorname{dom} F) \neq \emptyset$ by (17), and the last identity follows from Corollary 14, This establishes the desired inclusion and thus concludes the proof.

Of course, using Fermat's rule, i.e. $\operatorname{argmin} \Phi=(\partial \Phi)^{-1}(0)$, and the fact that $\inf \Phi=-\inf \Phi^{*}(0)$ for any convex function $\Phi$, Corollary [16] can be exploited to derive (necessary and sufficient) optimality conditions and a duality framework for the convex-composite optimization problem (11). We will do this explicitly in Section 5.1 for conic programming. General optimality conditions for the convex-composite problem (11) are derived in [44, Proposition 8.1 (ii)] under (17).

The same expressions for the conjugate and subdifferential as in Corollary 16 were derived by Combari et al. [22, Proposition 4.11] and Bot et al. [8, Theorem 3.3, Corollary 3.4]. The arguments (adaptable to the infinite-dimensional setting) used in [8, 22, are based on investigating properties of a suitable perturbation function and hence, they require different notions of lower semicontinuity of $F$ even for the finite-dimensional setting. Our arguments, on the other hand, are in essence a synthesis of the infimal convolution approach to convex-composite functions inspired by [32] and the $K$-convexity study in [44]. This allows us to eliminate the assumptions on lower semicontinuity of $f, g$ and $F$ and to relax the $K$-increasing property (8) of $g$ to the weaker condition (7).

The results by Bot et al. [8, Theorem 3.3, Corollary 3.4] show that for $f \in$ $\Gamma_{0}\left(\mathbb{E}_{1}\right), g \in \Gamma_{0}\left(\mathbb{E}_{2}\right) K$-increasing, and $\left\{\langle v, F\rangle \mid v \in-K^{\circ}\right\} \subset \Gamma_{0}\left(\mathbb{E}_{1}\right)$, the conjugate formula from Corollary [16 a) holds if and only if

$$
\bigcup_{v \in \operatorname{dom} g^{*}}\left(\left(0, g^{*}(v)\right)+\operatorname{epi}(f+\langle v, F\rangle)^{*}\right)
$$

is closed. The subdifferential formula from Corollary $16 \mathrm{~b}$ ) then follows. We point out that, although [8, Theorem 3.3] is a deep result, condition (22) is, in general, not verifiable. On the other hand, in the infinite dimensional setting its verifiability is comparable to the one for Attouch-Brézis-type conditions. 
The study by Combari et al. 22, like ours, provides verifiable conditions for the conjugate and subdifferential formulae. Therefore we would briefly like to discuss the differences in assumptions, in particular the qualification conditions, when compared in the finite-dimensional setting: In [22, the authors assume, as we do, the properness and $K$-convexity of all functions in play. However, they also need the assumption that $F$ is lower semicontinuous in a generalized sense, see [22, Definition 3.1], and they also assume $g$ to be $K$-increasing (8), whereas we only require the weaker assumption (77). We note that, under (8), our Slater-type condition (17) becomes

$$
F(\operatorname{ri}(\operatorname{dom} f) \cap \operatorname{ri}(\operatorname{dom} F)) \cap \operatorname{ri}(\operatorname{dom} g) \neq \emptyset .
$$

The (Attouch-Brézis-type) qualification condition comparable to ours, used in [22] reads

$$
\mathbb{R}_{+}(\operatorname{dom} g-F(\operatorname{dom} F \cap \operatorname{dom} f)) \text { is a subspace }
$$

which, using the fact that $\operatorname{dom} g=\operatorname{dom} g-K$ and $K+F(\operatorname{dom} F \cap \operatorname{dom} f)$ is convex, yields

$$
0 \in \operatorname{ri}(K+F(\operatorname{dom} f \cap \operatorname{dom} F)-\operatorname{dom} g)
$$

or, equivalently,

$$
\operatorname{ri}(K+F(\operatorname{dom} f \cap \operatorname{dom} F)) \cap \operatorname{ri}(\operatorname{dom} g) \neq \emptyset .
$$

This condition differs from (23) in general. When $f$ and $F$ are finite-valued and rge $F$ is convex then (24) becomes

$$
\operatorname{ri}(\operatorname{dom} g) \cap \operatorname{ri}(\operatorname{rge} F) \neq \emptyset \text {, }
$$

which is stronger than our condition, which then reads

$$
\text { ri }(\operatorname{dom} g) \cap \operatorname{rge} F \neq \emptyset \text {. }
$$

The formula for $(g \circ F)^{*}$ is also obtained in [5, Section 4] under the following qualification condition

$$
\text { ri }(\operatorname{rge} F \cap \operatorname{dom} g+K) \cap \operatorname{ri}(\operatorname{dom} g) \neq \emptyset,
$$

which, using the fact that $\operatorname{dom} g=\operatorname{dom} g-K$, is exactly equivalent to (25) if rge $F$ is convex. This qualification condition also appeared in [54, Theorem 2.8] where the author derived a duality result as well as the formula for $\varepsilon$-subdifferential for $g \circ F$.

The following example, and also Example 34, illustrates that (7) is, indeed, weaker than (8), and that our qualification condition (17) differs from, and is often weaker than (24).

Example 17. Let $\mathbb{E}_{1}=\mathbb{E}_{2}=\mathbb{R}^{2}, K=\mathbb{R}_{+} \times\{0\}, F:\left(x_{1}, x_{2}\right) \in \mathbb{E}_{1} \mapsto\left(0, x_{2}\right)$,

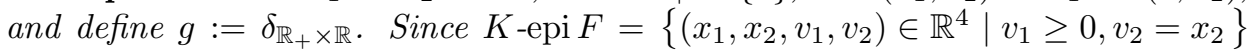
is convex, $F$ is $K$-convex. Now, if $\operatorname{rge} F \ni\left(u_{1}, u_{2}\right) \leq_{K}\left(v_{1}, v_{2}\right)$, i.e., $0=u_{1} \leq$ $v_{1}, \quad u_{2}=v_{2}$, then $g\left(u_{1}, u_{2}\right)=g\left(v_{1}, v_{2}\right)$. This means that $g$ satisfies (7) but not (8) by taking $u_{1}<0$ and $v_{1} \geq 0$. Furthermore, we note that $\mathbb{R}_{+}(\operatorname{dom} g-\operatorname{rge} F)=\mathbb{R}_{+} \times \mathbb{R}$ is not a subspace, so (24) is violated, but (17) is satisfied as $\operatorname{ri}(\operatorname{dom} g-K) \cap \operatorname{rge} F=$ $\mathbb{R}^{2} \cap\{0\} \times \mathbb{R} \neq \emptyset$.

The next corollary follows simply from the fact that condition (7) is trivially satisfied if $g$ is $K$-increasing and that (10) then simplifies to

$$
F(\operatorname{ri}(\operatorname{dom} F)) \cap \operatorname{ri}(\operatorname{dom} g) \neq \emptyset \text {. }
$$


Corollary 18. Let $K \subset \mathbb{E}_{2}$ be a closed, convex cone, $F: \mathbb{E}_{1} \rightarrow \mathbb{E}_{2}^{\bullet} K$-convex, $g \in \Gamma$ and $K$-increasing such that (26) holds (which is true in particular when $g$ is finite-valued or $F$ is surjective and $\left.\operatorname{dom} F=\mathbb{E}_{2}\right)$. Then

$$
(g \circ F)^{*}(p)=\min _{v \in-K^{\circ}} g^{*}(v)+\langle v, F\rangle^{*}(p)
$$

and

$$
\partial(g \circ F)(\bar{x})=\bigcup_{v \in \partial g(F(\bar{x}))} \partial\langle v, F\rangle(\bar{x}) \quad(\bar{x} \in \operatorname{dom} g \circ F) .
$$

Proof. Since the fact that $g$ is $K$-increasing implies (7D) the assertion follows from Theorem 12 and Corollary [14, respectively.

The next result shows that any closed, proper, convex function $g$ is increasing with respect to -hzn $g$.

Lemma 19. Let $g \in \Gamma_{0}$. Then $g$ is (-hzn $\left.g\right)$-increasing.

Proof. Put $K:=-$ hzn $g$. Then [47, Theorem 14.2] yields $K=-\left(\overline{\operatorname{cone}}\left(\operatorname{dom} g^{*}\right)\right)^{\circ}$. Now let $x, y \in \mathbb{E}$ such that $x \preceq_{K} y$, i.e. $y=x+b$ for some $b \in K$. Since $g=g^{* *}$ we hence find

$$
\begin{aligned}
g(x) & =\sup _{z \in \operatorname{dom} g^{*}}\left\{\langle x, z\rangle-g^{*}(z)\right\} \\
& =\sup _{z \in \operatorname{dom} g^{*}}\left\{\langle y, z\rangle-\langle b, z\rangle-g^{*}(z)\right\} \\
& \leq \sup _{z \in \operatorname{dom} g^{*}}\left\{\langle y, z\rangle-g^{*}(z)\right\} \\
& =g(y),
\end{aligned}
$$

where the inequality relies on the fact that $\langle b, z\rangle \geq 0$.

The observation from Lemma 19 that any closed, proper, convex function is increasing with respect to its own negative horizon cone brings us back full circle to the starting point of our study in Theorem 1. The latter theorem is now merely an immediate consequence of Lemma 19 and Proposition 7 , and we observe that $F$ can even be extended-valued. We pursue this setting where $g$ is closed, proper, convex and $F$ is convex with respect to the negative horizon cone of $g$ in the next result.

Corollary 20. Let $g \in \Gamma_{0}\left(\mathbb{E}_{2}\right)$ and let $F: \mathbb{E}_{1} \rightarrow \mathbb{E}_{2}^{\bullet}$ be $(-\mathrm{hzn} g)$-convex such that

$$
F(\operatorname{ri}(\operatorname{dom} F)) \cap \operatorname{ri}(\operatorname{dom} g) \neq \emptyset \text {. }
$$

Then

$$
(g \circ F)^{*}(p)=\min _{v \in \mathbb{E}_{2}} g^{*}(v)+\langle v, F\rangle^{*}(p)
$$

and

$$
\partial(g \circ F)(\bar{x})=\bigcup_{v \in \partial g(F(\bar{x}))} \partial\langle v, F\rangle(\bar{x}) \quad(\bar{x} \in \operatorname{dom} g \circ F) .
$$

Proof. This follows from combining Corollary 18 (with $K=-$ hzn $g$ ) and Lemma 19 while observing that $\operatorname{dom} g^{*} \subset \overline{\operatorname{cone}}\left(\operatorname{dom} g^{*}\right)=(\text { hzn } g)^{\circ}$, cf. [47, Theorem 14.2].

The next corollary covers the setting considered in 32 where $F$ is a componentwise convex function, and whose technique of proof served as a source of inspiration for our study. 
Corollary 21. Let $g \in \Gamma\left(\mathbb{R}^{m}\right)$ be $\mathbb{R}_{+}^{m}$-increasing, $F: \mathbb{E} \rightarrow\left(\mathbb{R}^{m}\right)^{\bullet}$ with $F_{i} \in$ $\Gamma(\mathbb{E})(i=1, \ldots, m)$ such that

$$
F\left(\bigcap_{i}^{m} \operatorname{ri}\left(\operatorname{dom} F_{i}\right)\right) \cap \operatorname{ri}(\operatorname{dom} g) \neq \emptyset .
$$

Then

$$
(g \circ F)^{*}(p)=\min _{v \geq 0} g^{*}(v)+\left(\sum_{i=1}^{m} v_{i} F_{i}\right)^{*}(p)
$$

and

$$
\partial(g \circ F)(\bar{x})=\bigcup_{v \in \partial g(F(\bar{x}))} \sum_{i=1}^{m} v_{i} \partial F_{i}(\bar{x}) \quad(\bar{x} \in \operatorname{dom} g \circ F) .
$$

Proof. Apply Corollary 18 with $K=\mathbb{R}_{+}^{m}$ and observe that $\partial g(F(\bar{x})) \subset \mathbb{R}_{+}^{m}$, cf. Lemma $13 \mathrm{a})$, hence $\partial\langle v, F\rangle(\bar{x})=\sum_{i=1}^{m} v_{i} \partial F_{i}(\bar{x})$ for all $v \in g(F(\bar{x}))$.

Finally, as another immediate consequence of our study, we get the well-known result for the case when $F$ is linear.

Corollary 22 (The linear case). Let $g \in \Gamma\left(\mathbb{E}_{2}\right)$ and $F: \mathbb{E}_{1} \rightarrow \mathbb{E}_{2}$ linear such that rge $F \cap \operatorname{ri}(\operatorname{dom} g) \neq \emptyset$. Then

$$
(g \circ F)^{*}(p)=\min _{v \in \mathbb{E}_{2}}\left\{g^{*}(v) \mid F^{*}(v)=p\right\}
$$

with $\operatorname{dom}(g \circ F)=\left(F^{*}\right)^{-1}\left(\operatorname{dom} g^{*}\right)$, and

$$
\partial(g \circ F)(\bar{x})=F^{*}(\partial g(F(\bar{x}))) \quad(\bar{x} \in \operatorname{dom} g \circ F) .
$$

Proof. We notice that $F$ is $\{0\}$-convex. Hence we can apply Corollary 18 with $K=\{0\}$. Condition (26) then reads rge $F \cap \operatorname{ri}(\operatorname{dom} g) \neq \emptyset$, which is our assumption. Hence we obtain

$$
(g \circ F)^{*}(p)=\min _{v \in-K^{\circ}} g^{*}(v)+\langle v, F\rangle^{*} p=\min _{v \in \mathbb{E}_{2}} g^{*}(v)+\delta_{F^{*}(v)}(p) .
$$

where the second identity uses Lemma 6. Moreover, $\partial\langle v, F\rangle(\bar{x})=F^{*}(v)$ for all $v \in \mathbb{E}_{2}$, which proves the subdifferential formula.

\section{Applications}

In this section we present an eclectic series of applications of our study in Section 3 and 4 to illustrate the versatility of our findings and to establish connections to different areas of convex analysis, optimization and matrix analysis.

5.1 Conic programming We consider the general conic program [51]

$$
\min f(x) \text { such that } F(x) \in-K
$$

where $f \in \Gamma\left(\mathbb{E}_{1}\right), F: \mathbb{E}_{1} \rightarrow \mathbb{E}_{2}$ is $K$-convex and $K \subset \mathbb{E}_{2}$ is a closed, convex cone. Clearly, (27) can be written in the additive composite form

$$
\min _{x \in \mathbb{E}_{1}} f(x)+\left(\delta_{-K} \circ F\right)(x) .
$$

This fits the additive composite setting of Corollary 16 with $g=\delta_{-K}$ which is $K$-increasing (in fact, let $u \leq_{K} v$, if $v \in-K$ then $u=u-v+v \in-K-K=-K$ 
and hence, in all cases, $g(u) \leq g(v)$ ). Moreover, the qualification condition (17) reads

$$
F(\operatorname{ri}(\operatorname{dom} f)) \cap \operatorname{ri}(-K) \neq \emptyset .
$$

The Fenchel(-Rockafellar) dual problem associated with (27) via (28) is

$$
\max _{y \in \mathbb{E}_{1}}-f^{*}(y)-\left(\delta_{-K} \circ F\right)^{*}(-y),
$$

while the Lagrangian dual is

$$
\max _{v \in-K^{\circ}} \inf _{x \in \mathbb{E}_{1}} f(x)+\langle v, F(x)\rangle .
$$

We obtain the following duality result.

Theorem 23 (Strong duality and dual attainment for conic programming). Let $f \in \Gamma\left(\mathbb{E}_{1}\right), K \subset \mathbb{E}_{2}$ a closed, convex cone, and let $F: \mathbb{E}_{1} \rightarrow \mathbb{E}_{2}$ be $K$-convex. If (29) holds then

$$
\begin{aligned}
\inf _{x \in \mathbb{E}_{1}} f(x)+\left(\delta_{-K} \circ F\right)(x) & =\max _{v \in-K^{\circ}}-f^{*}(y)-\left(\delta_{-K} \circ F\right)^{*}(-y) \\
& =\max _{v \in-K^{\circ}} \inf _{x \in \mathbb{E}_{1}} f(x)+\langle v, F(x)\rangle .
\end{aligned}
$$

Proof. Observe that, by Corollary [16, we have

$$
\begin{aligned}
\left(f+\delta_{-K} \circ F\right)^{*}(0) & =\min _{\substack{v \in-K^{\circ} \\
y \in \mathbb{E}_{1}}} f^{*}(y)+\langle v, F\rangle^{*}(-y) \\
& =\min _{v \in-K^{\circ}}(f+\langle v, F\rangle)^{*}(0) \\
& =\min _{v \in-K^{\circ}} \sup _{x \in \mathbb{E}_{1}}\{-f(x)-\langle v, F(x)\rangle\} .
\end{aligned}
$$

Moreover, by Theorem 12 we have

$$
\left(\delta_{-K} \circ F\right)^{*}(-y)=\min _{v \in-K^{\circ}}\langle v, F\rangle^{*}(-y) .
$$

Finally, since $\inf _{\mathbb{E}_{1}} f+\delta_{-K} \circ F=-\left(f+\delta_{-K} \circ F\right)^{*}(0)$, this shows everything.

Theorem 23 furnishes various facts: It shows strong duality and dual attainment for both the Fenchel duality (first identity) and Lagrangian duality (second identity) scheme under a generalized Slater condition. Moreover, it shows the equivalence of both duality concepts. Of course, these are well-known results, but the proof based on Corollary 16 and Theorem [12, respectively, unifies this in a very elegant and convenient way.

Optimality conditions are also easily derived. Here observe that for a convex set $C \subset \mathbb{E}$, the normal cone of $C$ at $\bar{x} \in C$ is

$$
N_{C}(\bar{x})=\partial \delta_{C}(\bar{x})=\{v \mid\langle v, x-\bar{x}\rangle \leq 0(x \in C)\} .
$$

Theorem 24. Let $f \in \Gamma\left(\mathbb{E}_{1}\right), K \subset \mathbb{E}_{2}$ a closed, convex cone, and let $F: \mathbb{E}_{1} \rightarrow \mathbb{E}_{2}$ be $K$-convex. Then the condition

$$
0 \in \partial f(\bar{x})+\bigcup_{v \in N_{-K}(F(\bar{x}))} \partial\langle v, F\rangle(\bar{x})
$$

is sufficient for $\bar{x}$ to be a minimizer of (27). Under (29) it is also necessary.

Proof. From Corollary 16 b) with $g=\delta_{-K}$, (30) it follows that $0 \in \partial(f+g \circ F)(\bar{x})$, so $\bar{x}$ is a minimizer of $f+g \circ F$. Under (29), again by Corollary $16 \mathrm{~b}), \partial(f+g \circ F)(\bar{x})$ equals the set on the right-hand side of (30), which concludes the proof. 
The differentiable case merits its own statement.

Corollary 25. Let $f: \mathbb{E}_{1} \rightarrow \mathbb{R}$ be differentiable and convex, $K \subset \mathbb{E}_{2}$ a closed, convex cone, and let $F: \mathbb{E}_{1} \rightarrow \mathbb{E}_{2}$ be differentiable and $K$-convex. Then the condition

$$
-\nabla f(\bar{x}) \in F^{\prime}(\bar{x})^{*} N_{-K}(F(\bar{x}))
$$

is sufficient for $\bar{x}$ to be a minimizer of (27). Under the condition

$$
\text { rge } F \cap \operatorname{ri~}(-K) \neq \emptyset
$$

it is also necessary.

Proof. This follows immediately from Theorem 24, Remark 15] and the fact that (29) reduces to (32) when $\operatorname{dom} f=\mathbb{E}_{1}$.

5.2 The pointwise maximum of convex functions In what follows we denote the unit simplex in $\mathbb{R}^{m}$ by $\Delta_{m}$, i.e.

$$
\Delta_{m}=\left\{v \in \mathbb{R}^{m} \mid v_{i} \geq 0, \sum_{i=1}^{m} v_{i}=1\right\} .
$$

The following result provides the conjugate formula for the pointwise maximum of finitely many convex functions. It therefore slightly generalizes (at least in the finite dimensional case) the results established for the case of two functions in 31] and alternatively proven in [6]. The well-known subdifferential formula is also derived.

Proposition 26. For $f_{1}, \ldots, f_{m} \in \Gamma_{0}(\mathbb{E})$ define $f:=\max _{i=1, \ldots, m} f_{i}$. Then $f \in \Gamma_{0}(\mathbb{E})$ with $\operatorname{dom} f=\bigcap_{i=1}^{m} \operatorname{dom} f_{i}$,

$$
f^{*}(x)=\min _{v \in \Delta_{m}}\left(\sum_{i=1}^{m} v_{i} f_{i}\right)^{*}(x)
$$

and

$$
\partial f(\bar{x})=\operatorname{conv}\left\{\partial f_{i}(\bar{x}) \mid i \in I(\bar{x})\right\},
$$

where $I(\bar{x})=\left\{i \mid f_{i}(\bar{x})=f(\bar{x})\right\}$.

Proof. Define $F: \mathbb{E} \rightarrow\left(\mathbb{R}^{m}\right)^{\bullet}$ by

$$
F(x)= \begin{cases}\left(f_{1}(x), \ldots, f_{m}(x)\right) & \text { if } x \in \bigcap_{i=1}^{m} \operatorname{dom} f_{i}, \\ +\infty & \text { otherwise, }\end{cases}
$$

and $g: \mathbb{R}^{m} \rightarrow \mathbb{R}$ by $g(v)=\max _{i=1, \ldots, m} v_{i}$. Then $f=g \circ F$ (with the conventions made in Section 21) and we observe that $F$ is $\mathbb{R}_{+}^{m}$-convex and $g$ is $\mathbb{R}_{+}^{m}$-increasing with $\operatorname{dom} g=\mathbb{R}^{m}$. Hence, Corollary 18 is applicable with the qualification condition (10) trivially satisfied. Thus, for all $x \in \mathbb{E}$, we obtain

$$
\begin{aligned}
(g \circ F)^{*}(x) & =\min _{v \in \mathbb{R}_{+}^{m}} g^{*}(v)+\langle v, F\rangle^{*}(x) \\
& =\min _{v \in \mathbb{R}_{+}^{m}} \delta_{\Delta_{m}}(v)+\langle v, F\rangle^{*}(x) \\
& =\min _{v \in \Delta_{m}}\left(\sum_{i=1}^{m} v_{i} f_{i}\right)^{*}(x)
\end{aligned}
$$


where the second equality follows from [1, Example 4.10]. Moreover

$$
\begin{aligned}
\partial f(\bar{x}) & =\bigcup_{v \in \partial g(F(\bar{x}))} \partial\langle v, F\rangle(\bar{x}) \\
& =\bigcup_{v \in \operatorname{conv}\left\{e_{i} \mid\right.} \sum_{i \in I(\bar{x})\}}^{m} v_{i} \partial f_{i}(\bar{x}) \\
& =\operatorname{conv}\left\{\partial f_{i}(\bar{x}) \mid i \in I(\bar{x})\right\} .
\end{aligned}
$$

Here the second identity uses the known fact that $\partial g(y)=\operatorname{conv}\left\{e_{i} \mid y_{i}=g(y)\right\}$ for all $y \in \mathbb{R}^{m}$.

\subsection{Kiefer-Gaffke-Krafte inequality and the matrix-fractional function} Recall that $\mathbb{C}^{n \times m}$ and $\mathbb{H}^{n}$ are the linear spaces of complex $n \times m$ and Hermitian $n \times n$ matrices, respectively. Consider the Euclidean space

$$
\mathbb{G}:=\mathbb{C}^{n \times m} \times \mathbb{H}^{n}
$$

equipped with the inner product

$$
\langle\cdot, \cdot\rangle:((X, U),(Y, V)) \in \mathbb{G} \times \mathbb{G} \mapsto \operatorname{Re} \operatorname{tr}\left(Y^{*} X\right)+\operatorname{Re} \operatorname{tr}(V U) .
$$

Define the mapping $F: \mathbb{G} \rightarrow\left(\mathbb{H}^{n}\right)^{\bullet}$ by

$$
F(X, V):=\left\{\begin{array}{rc}
X^{*} V^{\dagger} X & \text { if } \quad \operatorname{rge} X \subset \operatorname{rge} V \\
+\infty & \text { else, }
\end{array}\right.
$$

where $X^{*}$ is the adjoint of $X$ and $V^{\dagger}$ is the Moore-Penrose pseudoinverse of $V$, cf. [35]. Kiefer [38 has shown that $F$ is $\mathbb{H}_{+}^{n}$-convex on the set

$$
\mathcal{Q}:=\left\{(X, V) \in \mathbb{C}^{n \times m} \times \mathbb{H}_{++}^{n} \mid \operatorname{rge} X \subset \operatorname{rge} V\right\},
$$

and that

$$
\begin{array}{cc} 
& F\left(\sum_{i=1}^{k} \lambda_{i}\left(X_{i}, V_{i}\right)\right)=\sum_{i=1}^{k} \lambda_{i} F\left(X_{i}, V_{i}\right) \quad\left(\lambda \in \Delta_{k},\left(X_{i}, V_{i}\right) \in \mathcal{Q}\right) \\
& X_{i}^{*} V_{i}^{-1}=X_{i}^{*} V_{j}^{-1} \quad(i, j \in\{1, \ldots, k\}) .
\end{array}
$$

Gaffke and Krafte 34] extend this result by showing that $F$ is convex on the set

$$
\operatorname{cl} \mathcal{Q}=\left\{(X, V) \in \mathbb{C}^{n \times m} \times \mathbb{H}_{+}^{n} \mid \operatorname{rge} X \subset \operatorname{rge} V\right\}
$$

again with respect to the cone $\mathbb{H}_{+}^{n}$, and (36) holds for a convex combination $\sum_{i=1}^{k} \lambda_{i}\left(X_{i}, V_{i}\right)$ of points in $\mathrm{cl} \mathcal{Q}$ if and only if there is a matrix $C \in \mathbb{C}^{n \times m}$ such that $X_{i}=V_{i} C$ for $i=1, \ldots, k$.

The $\mathbb{H}_{+}^{n}$-convexity of the mapping $F$ from (35) is closely tied to the matrixfractional function [15, 16, 18, $\gamma: \widehat{\mathbb{G}} \rightarrow \mathbb{R} \cup\{+\infty\}$ given by

$$
\gamma(X, V):=\left\{\begin{aligned}
\frac{1}{2} \operatorname{tr}(F(X, V)), & \text { if } \quad(X, V) \in \widehat{\mathcal{Q}}, \\
+\infty, & \text { else, }
\end{aligned}\right.
$$

where $\widehat{\mathbb{G}}: \mathbb{R}^{n \times m} \times \mathbb{S}^{n}$ and $\widehat{\mathcal{Q}}:=\left\{(X, V) \in \widehat{\mathbb{G}} \mid V \in \mathbb{S}_{+}^{n}\right.$, rge $\left.X \subset \operatorname{rge} V\right\}$. In [18, Section 5.2], it is shown that $\gamma$ is the support function of the set

$$
\mathcal{F}:=\left\{\left(Y,-\frac{1}{2} Y Y^{T}\right) \mid Y \in \mathbb{R}^{n \times m}\right\}
$$


and so $\gamma$ is a closed, proper, convex (even sublinear) function with domain $\widehat{\mathcal{Q}}$. However, Theorem 5 can be used to establish a new and stronger result concerning the convexity of $\gamma$.

Proposition 27. Let $\mathbb{G}$ be given by (33), $F: \mathbb{G} \rightarrow\left(\mathbb{H}^{n}\right)^{\bullet}$ by (35) and define $\tilde{\gamma}: \mathbb{G} \rightarrow \mathbb{R} \cup\{+\infty\}$ by

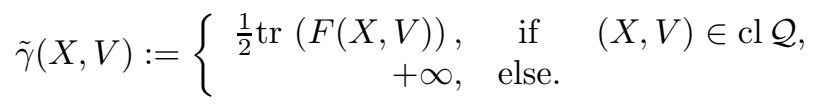

Then $\tilde{\gamma}$ is a support function, in particular closed, proper, and convex.

Proof. Let $\mathbb{E}_{1}:=\mathbb{G}$ and $\mathbb{E}_{2}:=\mathbb{H}^{n}$ equipped with the inner products based on (34) and set $g:=\left\langle\frac{1}{2} I, \cdot\right\rangle \in \Gamma\left(\mathbb{E}_{2}\right)$. Then $\tilde{\gamma}=g \circ F$. Since $\frac{1}{2} I \in \mathbb{H}_{++}^{n}=-\left(\mathbb{H}_{++}^{n}\right)^{\circ}$, Theorem [5] tells us that $\tilde{\gamma}$ is convex. Obviously $\tilde{\gamma}$ is also proper and positively homogeneous, i.e. $\tilde{\gamma}(\alpha(X, V))=\alpha \tilde{\gamma}(X, V)$ for any $\alpha \geq 0$. We now show that it is closed: To this end, let $\left\{\left(\left(X_{k}, V_{k}\right), \mu_{k}\right)\right\} \subset$ epi $\tilde{\gamma}$ and $((\tilde{X}, \tilde{V}), \tilde{\mu}) \in \mathbb{G} \times \mathbb{R}$ be such that $\left(\left(X_{k}, V_{k}\right), \mu_{k}\right) \rightarrow((\widetilde{X}, \widetilde{V}), \tilde{\mu})$. We need to show that $((\tilde{X}, \widetilde{V}), \tilde{\mu}) \in$ epi $\tilde{\gamma}$. Let $V_{k}$ have reduced singular-value decomposition $V_{k}=U_{k} D_{k} U_{k}^{*}$ for each $k \in \mathbb{N}$ so that $U_{k} \in \mathbb{C}^{n \times r_{k}}$ with $r_{k}:=\operatorname{rank} V_{k} \leq n, U_{k}^{*} U_{k}=I_{r_{k}}$ and $D_{k}=\operatorname{diag}\left(\sigma_{k 1}, \ldots, \sigma_{k r_{k}}\right)$ with $\sigma_{k j} \in \mathbb{R}_{++}$for $j=1, \ldots, r_{k}$ and all $k \in \mathbb{N}$. Set $\tilde{r}:=\operatorname{rank} \widetilde{V}$. With no loss in generality, there is a $r \in\{\tilde{r}, \tilde{r}+1, \ldots, n\}$ and $\widetilde{U} \in \mathbb{C}^{n \times r}$ such that $r_{k}=r$ for all $k \in \mathbb{N}$ with $\widetilde{U}^{*} \widetilde{U}=I_{r}$ and $U_{k} \rightarrow \widetilde{U}$. Then $D_{k}=U_{k}^{*} V_{k} U_{k} \rightarrow \widetilde{U}^{*} \widetilde{V} \widetilde{U}=: \widetilde{D}=$ $\operatorname{diag}\left(\sigma_{1}, \ldots, \sigma_{r}\right)$ for some $\sigma_{j} \in \mathbb{R}_{+}, j=1, \ldots, r$ satisfying $\sigma_{k j} \rightarrow \sigma_{j}, j=1, \ldots, r$. Since rge $X_{k} \subset \operatorname{rge} V_{k}$, there exists $C_{k} \in \mathbb{C}^{r \times m}$ such that $X_{k}=U_{k} C_{k}$, for all $k \in \mathbb{N}$, with $C_{k}=U_{k}^{*} X_{k} \rightarrow \widetilde{U}^{*} \widetilde{X}=: \widetilde{C}$. Denote the columns of $C_{k}^{*}$ by $c_{k j}, j=1, \ldots, r, k \in$ $\mathbb{N}$, and those of $\widetilde{C}^{*}$ by $\tilde{c}_{j}, j=1, \ldots, r$. Then

$$
0 \leq \sum_{j=1}^{r} \sigma_{k j}^{-1}\left\|c_{k j}\right\|_{2}^{2}=\operatorname{tr}\left(C_{k}^{*} D_{k}^{-1} C_{k}\right)=2 \tilde{\gamma}\left(X_{k}, V_{k}\right) \leq 2 \mu_{k}
$$

Consequently,

$$
0 \leq \limsup _{k \rightarrow \infty} \sum_{j=1}^{r} \sigma_{k j}^{-1}\left\|c_{k j}\right\|_{2}^{2} \leq 2 \tilde{\mu} .
$$

Hence, by passing to a subsequence if necessary, we can assume that there exists $\eta_{j} \in \mathbb{R}_{+}$such that $\sigma_{k j}^{-1}\left\|c_{k j}\right\|_{2}^{2} \rightarrow \eta_{j}$ for $j=1, \ldots, r$. Observe that if, for some $j_{0} \in\{1, \ldots, r\}, \sigma_{k j} \rightarrow \underset{\widetilde{V}}{0}$, then it must be the case that $c_{k j_{0}} \rightarrow 0$ since $\eta_{j_{0}} \in \mathbb{R}_{+}$. Therefore, rge $\widetilde{X} \subset \operatorname{rge} \widetilde{V}$, so that $(\tilde{X}, \widetilde{V}) \in \operatorname{dom} \tilde{\gamma}$ with

$$
2 \tilde{\gamma}(\tilde{X}, \widetilde{V})=\sum_{j=1}^{r} \sigma_{j}^{\dagger}\left\|\tilde{c}_{j}\right\|_{2}^{2} \leq \sum_{j=1}^{r} \eta_{j} \leq 2 \tilde{\mu}
$$

That is, $\tilde{\gamma}$ is closed. Here $\sigma_{j}^{\dagger}=\sigma_{j}^{-1}$ if $\sigma_{j}>0$ and 0 otherwise.

All in all, we have shown that $\tilde{\gamma}$ is closed, properm convex and positively homogeneous. The claim therefore follows from Hörmander's Theorem, see e.g. [47. Corollary 13.2.1]. 
5.4 Variational Gram functions We equip $\mathbb{S}^{n}$ with the inner product $\langle X, Y\rangle=$ $\operatorname{tr}(X Y)$. Given a set $M \subset \mathbb{S}_{+}^{n}$, the associated variational Gram function (VGF) [16, 36] is given by

$$
\Omega_{M}: \mathbb{R}^{n \times m} \rightarrow \mathbb{R} \cup\{+\infty\}, \quad \Omega_{M}(X)=\frac{1}{2} \sigma_{M}\left(X X^{T}\right) .
$$

Since $\sigma_{M}=\sigma_{\overline{\mathrm{conv}} M}$ there is no loss in generality to assume that $M$ is closed and convex. For the remainder of this paragraph we let

$$
F: \mathbb{R}^{n \times m} \rightarrow \mathbb{S}^{n}, \quad F(X)=\frac{1}{2} X X^{T} .
$$

Then $\Omega_{M}=\sigma_{M} \circ F$ fits the composite scheme studied in Sections 34 It is obvious that $\operatorname{rge} F=\mathbb{S}_{+}^{n}$. The following lemma clarifies the $K$-convexity properties of $F$.

Lemma 28. Let $F$ be given by (40). Then $\mathbb{S}_{+}^{n}$ is the smallest closed, convex cone in $\mathbb{S}^{n}$ with respect to which $F$ is convex.

Proof. Let $K$ be the smallest closed convex cone in $\mathbb{S}^{n}$ such that $F$ is $K$-convex. On the one hand, it is easily verified that $F$ is $\mathbb{S}_{+}^{n}$-convex, hence $K \subset \mathbb{S}_{+}^{n}$. On the other hand, by [44, Lemma 6.1],

$$
(-K)^{\circ}=K_{F}:=\left\{V \in \mathbb{S}^{n} \mid\langle V, F\rangle \text { is convex }\right\} .
$$

Now fixing $V \in \mathbb{S}^{n}$ for all $X \in \mathbb{R}^{n \times m}$ the mapping $\nabla^{2}\langle V, F\rangle(X): \mathbb{R}^{n \times m} \times \mathbb{R}^{n \times m} \rightarrow$ $\mathbb{R}$ is given by

$$
\nabla^{2}\langle V, F\rangle(X)[D, H]=\left\langle V, \frac{1}{2}\left(H D^{T}+D H^{T}\right)\right\rangle .
$$

Clearly, this symmetric bilinear form is positive semidefinite if and only if $V \in \mathbb{S}_{+}^{n}$, which proves that $K_{F}=\mathbb{S}_{+}^{n}$. Finally, by taking the polar and using the fact that $\left(\mathbb{S}_{+}^{n}\right)^{\circ}=-\mathbb{S}_{+}^{n}$ we get

$$
K=-(-K)^{\circ}=-K_{F}^{\circ}=-\left(\mathbb{S}_{+}^{n}\right)^{\circ}=\mathbb{S}_{+}^{n} .
$$

We now verify the remaining conditions necessary to apply our convex-composite framework from Section 4 to $g:=\sigma_{M}$ and $F$ given by (40).

Corollary 29. Let $M \subset \mathbb{S}_{+}^{n}$ be nonempty, closed and convex, and let $F$ be given by (40). Then the following hold:

a) $-\operatorname{hzn} \sigma_{M} \supset \mathbb{S}_{+}^{n}$.

b) $\sigma_{M}$ is $\mathbb{S}_{+}^{n}$ increasing.

c) $M$ is bounded if and only if

$$
\text { rge } F \cap \operatorname{ri}\left(\operatorname{dom} \sigma_{M}\right) \neq \emptyset \text {. }
$$

Proof. a) We have cone $M \subset \mathbb{S}_{+}^{n}$ as $M \subset \mathbb{S}_{+}^{n}$, and hence, by [47, Theorem 14.2]

$$
- \text { hzn } \sigma_{M}=-(\overline{\text { cone }} M)^{\circ} \supset-\left(\mathbb{S}_{+}^{n}\right)^{\circ}=\mathbb{S}_{+}^{n} .
$$

b) This follows from a) and Corollary 19 .

c) Since $M^{\infty} \subset \mathbb{S}_{+}^{n}, M^{\infty}$ is pointed, hence by [50, Exercise 6.22], we find that

$$
\emptyset \neq \operatorname{int}\left[\left(M^{\infty}\right)^{\circ}\right]=\left\{W \in \mathbb{S}^{n} \mid \operatorname{tr}(W V)<0\left(V \in M^{\infty} \backslash\{0\}\right)\right\} .
$$

Hence, by [47, Corollary 14.2.1], we have

$$
\operatorname{ri}\left(\operatorname{dom} \sigma_{M}\right)=\operatorname{ri}\left[\left(M^{\infty}\right)^{\circ}\right]=\operatorname{int}\left[\left(M^{\infty}\right)\right]^{\circ}
$$


As $\operatorname{rge} F=\mathbb{S}_{+}^{n}$, condition (41) is equivalent to

$$
\mathcal{F}:=\left\{W \in \mathbb{S}_{+}^{n} \mid \operatorname{tr}(V W)<0\left(V \in M^{\infty} \backslash\{0\}\right)\right\} \neq \emptyset .
$$

We claim that

$$
\mathcal{F}:= \begin{cases}\mathbb{S}_{+}^{n}, & \text { if } M \text { is bounded } \\ \emptyset, & \text { else. }\end{cases}
$$

The first case is clear, since $M^{\infty}=\{0\}$ if (and only if) $M$ is bounded [50, Theorem 3.5], in which case the condition restricting $\mathcal{F}$ is vacuous.

On the other hand, if $M$ is unbounded, then there exists $V \in M^{\infty} \backslash\{0\} \subset \mathbb{S}_{+}^{n} \backslash\{0\}$. But then $\operatorname{tr}(V W) \geq 0$ for all $W \in \mathbb{S}_{+}^{n}$, see e.g. [35. Theorem 7.5.4], which proves the second case.

All in all, we have established the desired equivalence.

We now combine our analysis in Section 3 and 4 with the matrix-fractional function $\gamma$ from (38) to find a very short proof of the conjugate function $\Omega_{M}^{*}$ in case $M$ is bounded (hence compact). Here we note that for $K:=\mathbb{S}_{+}^{n}$ and

we have

$$
\Omega:=\left\{(Y, W) \in \mathbb{R}^{n \times m} \times \mathbb{S}^{n} \mid \frac{1}{2} Y Y^{T}+W \preceq 0\right\}
$$

$$
\sigma_{K \text {-epi } F}(X,-V)=\sigma_{\Omega}(X, V) \quad\left((X, V) \in \mathbb{R}^{n \times m} \times \mathbb{S}^{n}\right) .
$$

Corollary 29 c) shows that our framework does not apply when $M$ is unbounded as the crucial condition (10) is violated then.

The next result covers what was proven in [36, Proposition 3.4] entirely and one case of [16, Proposition 5.10].

Proposition 30. Let $M \subset \mathbb{S}_{+}^{n}$ be nonempty, convex and compact. Then $\Omega_{M}^{*}$ is finite-valued and given by

$$
\Omega_{M}^{*}(X)=\frac{1}{2} \min _{V \in M}\left\{\operatorname{tr}\left(X^{T} V^{\dagger} X\right) \mid \operatorname{rge} X \subset \operatorname{rge} V\right\} .
$$

Proof. $K:=\mathbb{S}_{+}^{n}$. Recall that $(\overline{\text { cone }} M)^{\circ}=\operatorname{hzn} \sigma_{M}$, see [47, Theorem 14.2]. Then $F$ given by (40) is $K$-convex by Lemma 28, and $g$ is $K$-increasing by Corollary 29 b). By Corollary 29 c) we find that condition (26) for $\sigma_{M} \circ F$ and $K$ is satisfied if (and only if) $M$ is bounded. Hence we compute that

$$
\begin{aligned}
\Omega_{M}^{*}(X) & =\min _{V \in-K^{\circ}} \delta_{M}(V)+\langle V, F\rangle^{*}(X) \\
& =\min _{V \in M} \sigma_{K \text {-epi } F}(X,-V) \\
& =\min _{V \in M} \sigma_{\Omega}(X, V) \\
& =\min _{V \in M} \gamma(X, V) \\
& =\min _{V \in M}\left\{\frac{1}{2} \operatorname{tr}\left(X^{T} V^{\dagger} X\right) \mid \operatorname{rge} X \subset \operatorname{rge} V\right\}
\end{aligned}
$$

Here the first identity is due to Corollary [18, the second is due to Lemma 6, the third uses (42), the fourth follows from [15, Theorem 2] and the last identity is simply the definition of $\gamma$ in (38).

As $M$ is compact this proves also the finite-valuedness and thus concludes the proof. 
A formula for the subdifferential of the VGF $\Omega_{M}$ is easily established as well.

Corollary 31. Let $M \subset \mathbb{S}_{+}^{n}$ be nonempty, convex and compact. Then

$$
\partial \Omega_{M}(X)=\left\{V X \mid V \in \underset{M}{\operatorname{argmax}}\left\langle X X^{T}, \cdot\right\rangle\right\} .
$$

Proof. First observe that $F^{\prime}(X)^{*} V=V X$ for all $X \in \mathbb{R}^{n \times m}$ and that $\partial \sigma_{M}(U)=$ $\operatorname{argmax}_{M}\langle U, \cdot\rangle$ for all $U \in \mathbb{S}^{n}$. Therefore, Corollary 18 (which is applicable for the same reasons as in Proposition 30) and Remark 15

5.5 Spectral function of symmetric matrices Consider the function

$$
\lambda: \mathbb{S}^{n} \rightarrow \mathbb{R}^{n}, \lambda(X):=\left(\lambda_{1}(X), \ldots, \lambda_{n}(X)\right), \quad\left(X \in \mathbb{S}^{n}\right)
$$

where $\lambda_{1}(X) \geq \ldots \geq \lambda_{n}(X)$ are the eigenvalues of $X$. In order to apply our framework from above, we equip $\mathbb{S}^{n}$ with the inner product

$$
\langle\cdot, \cdot\rangle: \mathbb{S}^{n} \times \mathbb{S}^{n} \rightarrow \mathbb{R},\langle X, Y\rangle=\operatorname{tr}(X Y)
$$

and $\mathbb{R}^{n}$ with the standard inner product. The following results are, even without any convexity assumptions, originally due to Lewis [40, 41] and were recently confirmed by a simplified proof due to Drusvyatskiy and Paquette [28, however under an additional lower semicontinuity assumption. Our technique of proof, based on the convex-composite framework with $g \in \Gamma\left(\mathbb{R}^{n}\right)$ and $F:=\lambda$, differs substantially from the latter references, but shares some similarity with [44, Section 9] and we make use of some of the auxiliary results established there. Note that the latter reference does not establish the conjugate formula below.

Proposition 32. Let $g \in \Gamma\left(\mathbb{R}^{n}\right)$ be permutation invariant (i.e., its value does not change by reordering of the argument vector) and let $\lambda: \mathbb{S}^{n} \rightarrow \mathbb{R}^{n}$ be given by (43). Then $g \circ \lambda$ is convex and

$$
(g \circ \lambda)^{*}=g^{*} \circ \lambda .
$$

Proof. Consider the closed, convex cone

$$
K=\left\{v \in \mathbb{R}^{n} \mid \sum_{i=1}^{k} v_{i} \geq 0, k=1, \ldots, n-1, \sum_{i=1}^{n} v_{i}=0\right\},
$$

whose polar cone is

$$
K^{\circ}=\left\{v \in \mathbb{R}^{n} \mid v_{1} \leq \ldots \leq v_{n}\right\} .
$$

By [47, Theorem 6.5] we have

$$
\operatorname{ri} K=\left\{v \in \mathbb{R}^{n} \mid \sum_{i=1}^{k} v_{i}>0, k=1, \ldots, n-1, \sum_{i=1}^{n} v_{i}=0\right\} .
$$

We first note that $\operatorname{dom} \lambda=\mathbb{S}^{n}$ and hence, $\lambda(\operatorname{ri}(\operatorname{dom} \lambda))=\operatorname{rge} \lambda=-K^{\circ}$. Next, by [44. Corollary 9.3], $\lambda$ is $K$-convex and for any $v \in-K^{\circ},\langle v, \lambda\rangle=\sigma_{\Omega_{v}}$ where $\Omega_{v}=\left\{X \in \mathbb{S}^{n} \mid v-\lambda(X) \in K\right\}$ is a closed, convex set in $\mathbb{S}^{n}$. In particular, $\lambda$ is $K$ convex, and $\langle v, \lambda\rangle^{*}=\delta_{\Omega_{v}}$. Moreover, observe that since $g$ is permutation invariant, so is $g^{*}$, and hence, by [44, Lemma 9.5], both $g$ and $g^{*}$ satisfy (7) with $F=\lambda$ and $K$ from (44), which shows in particular that $g \circ \lambda$ is convex, see Proposition 7 Now set $G:=\left\{v \in \mathbb{R}^{n} \mid v_{1}=\ldots=v_{n}\right\}$ and consider two cases: 
dom $g=G$ : For $X \in \mathbb{S}^{n}$ observe that

$$
\begin{aligned}
(g \circ \lambda)^{*}(X) & =\sup _{\lambda(Y) \in \operatorname{dom} g}\{\langle X, Y\rangle-g(\lambda(Y))\} \\
& =\sup _{\alpha \in \mathbb{R}}\left\{\left\langle X, \alpha I_{n}\right\rangle-g(\alpha, \ldots, \alpha)\right\} \\
& =\sup _{\alpha \in \mathbb{R}}\left\{\left\langle(\alpha, \ldots, \alpha),\left(\lambda_{1}(X), \ldots, \lambda_{n}(X)\right)\right\rangle-g(\alpha, \ldots, \alpha)\right\} \\
& =\sup _{y \in \operatorname{dom} g}\{\langle y, \lambda(X)\rangle-g(y)\} \\
& =g^{*}(\lambda(X)) .
\end{aligned}
$$

Here the second identity uses that $\operatorname{dom} g=G$ and that $\lambda(Y) \in G$ if and only if $Y$ is a multiple of $I_{n}$. The third is due to the fact that $\left\langle X, \alpha I_{n}\right\rangle=\alpha \operatorname{tr}(X)=$ $\alpha \sum_{i=1}^{m} \lambda_{i}(X)$. The fourth one uses again that $\operatorname{dom} g=G$. This proves the conjugate formula in this case.

$\operatorname{dom} g \neq G$ : Here we want to apply Theorem 12 to $g, F=\lambda$ and $K$ given in (44). We already established above that (7) holds. We now verify the qualification condition (10) which in the current setting reads

$$
\emptyset \neq \operatorname{ri}(\operatorname{dom} g-K) \cap\left(-K^{\circ}\right)=(\operatorname{ri}(\operatorname{dom} g)-\operatorname{ri} K) \cap\left(-K^{\circ}\right) .
$$

Fix $v \in \operatorname{ri}(\operatorname{dom} g)$. Denote by $S_{n}$ the set of all permutations $\sigma:\{1, \ldots, n\} \rightarrow$ $\{1, \ldots, n\}$ and the action of such a permutation on $v$ by $\sigma(v)=\left(v_{\sigma(1)}, \ldots, v_{\sigma(n)}\right)$. Since $g$ is unchanged under the reordering of its argument vector, $\sigma(v) \in \operatorname{dom} g$ for all $\sigma \in S_{n}$ and hence by [47, Theorem 6.1], $\bar{v}=\frac{1}{n !} \sum_{\sigma \in S_{n}} \sigma(v) \in \operatorname{ri}(\operatorname{dom} g)$. By assumption, $\operatorname{dom} g \neq G$, hence there exists $\hat{v} \in \operatorname{dom} g$ be such that at least two its components are distinct. Let $\sigma_{*} \in S_{n}$ be such that $\sigma_{*}(\hat{v})$ is a nondecreasing reordering of $\hat{v}$. By replacing $v$ by $\frac{1}{2} \bar{v}+\frac{1}{2} \sigma_{*}(\hat{v}) \in \operatorname{ri}(\operatorname{dom} g)$ (by [47, Theorem 6.1]), we can assume that $v_{1} \geq \ldots \geq v_{k}>v_{k+1} \geq \ldots \geq v_{n}$ for some $1 \leq k<n$. Now, pick $0<\alpha<\left(1-\frac{k}{n}\right)\left(v_{k}-v_{k+1}\right)$ and define $b \in \mathbb{R}^{n}$ component-wise by

$$
b_{i}=\left\{\begin{array}{ll}
\alpha, & \text { if } 1 \leq i \leq k, \\
-\frac{k \alpha}{n-k}, & \text { if } k<i \leq n
\end{array} \quad(i=1, \ldots, n) .\right.
$$

Then we have

$$
\sum_{i=1}^{\ell} b_{i}=k \cdot \alpha-\frac{\ell-k}{n-k} k \cdot \alpha \quad(\ell=1, \ldots, n),
$$

hence $b \in$ ri $K$, see (46). Moreover, we have

$\left(v_{i}-b_{i}\right)-\left(v_{i+1}-b_{i+1}\right)=\left\{\begin{array}{r}v_{k}-v_{k+1}-\frac{\alpha \cdot n}{n-k}, \\ v_{i}-v_{i+1}, \quad \text { if } \quad i=k,\end{array}\right\} \geq 0 \quad(i=1, \ldots, n)$, and therefore $v-b \in-K^{\circ}$, cf. (45). All in all, Theorem 12 is applicable and yields

$$
\begin{aligned}
(g \circ \lambda)^{*}(X) & =\min _{v \in-K^{\circ}} g^{*}(v)+\langle v, \lambda\rangle^{*}(X) \\
& =\min _{v \in-K^{\circ}} g^{*}(v)+\delta_{\Omega_{v}}(X) \\
& =\min _{v \in-K^{\circ}: X \in \Omega_{v}} g^{*}(v) \\
& =g^{*}(\lambda(X)),
\end{aligned}
$$

where the last identity follows from the fact that $-K^{\circ} \ni \lambda(X) \leq_{K} v$ and $g^{*}$ satisfies (77) as seen above. 
Remark 33. Using the same notation as in the proof of the Proposition 32, we have the following comments:

a) If $b=\left(b_{1}, \ldots, b_{n}\right) \in$ ri $K$ then $b_{n}<0<b_{1}$, and hence, we can deduce that the qualification condition (10) is equivalent to the fact that $\operatorname{dom} g \neq G$.

b) Analogous [40, 41, the formula for the subdifferential of $g \circ \lambda$ can be obtained using the conjugacy result in Proposition 32 and the von Neumann's inequality 43 .

$$
\langle X, Y\rangle \leq\langle\lambda(X), \lambda(Y)\rangle \quad\left(X, Y \in \mathbb{S}^{n}\right) .
$$

To this end, observe that

$$
\begin{aligned}
\partial(g \circ \lambda)(X) & =\left\{Y \in \mathbb{S}^{n} \mid(g \circ \lambda)(X)+(g \circ \lambda)^{*}(Y)=\langle X, Y\rangle\right\} \\
& =\left\{Y \in \mathbb{S}^{n} \mid g(\lambda(X))+g^{*}(\lambda(Y))=\langle\lambda(X), \lambda(Y)\rangle\right\} \\
& =\left\{Y \in \mathbb{S}^{n} \mid \lambda(Y) \in \partial g(\lambda(X))\right\} \\
& =\left\{U^{*} \operatorname{diag}(v) U \mid v \in \partial g(\lambda(X)), U^{*} U=I_{n}\right\},
\end{aligned}
$$

where the second equality comes from the Fenchel-Young inequality (3) applied to $g$.

We close this section with an example that shows that Proposition 32 cannot be derived from the results by Combari et al. [22] as their assumptions are not met.

Example 34. In Proposition [32, set $g=\delta_{\{0\}} \in \Gamma\left(\mathbb{R}^{n}\right)$ which is permutation invariant and satisfies (7) but is not increasing w.r.t. $K$ given in (44). Moreover, $\mathbb{R}_{+}(\operatorname{dom} g-\operatorname{rge} \lambda)=K^{\circ}$ is not a subspace.

5.6 A Farkas-type result In this section, we extend the Farkas-type result shown in [4, Theorem 4.1]. The latter reference uses a Lagrangian duality framework where our proof is based on Corollary 16] and Theorem 12. For this purpose, let $X \subset \mathbb{E}_{1}$ be a nonempty, convex set, let $K, L \subset \mathbb{E}_{2}$ be nonempty, closed, convex cones, let $F: \mathbb{E}_{1} \rightarrow \mathbb{E}_{2}^{\bullet}$ be $K$-convex, let $G: \mathbb{E}_{1} \rightarrow \mathbb{E}_{2}^{\bullet}$ be $L$-convex, $f \in \Gamma\left(\mathbb{E}_{1}\right)$, and let $g \in \Gamma\left(\mathbb{E}_{2}\right)$ satisfy (7). Consider the following qualification condition:

$$
\exists \bar{x} \in \operatorname{ri} X \cap \operatorname{ri}(\operatorname{dom} f) \cap \operatorname{ri}(\operatorname{dom} F) \cap \operatorname{ri}(\operatorname{dom} G): \quad\left\{\begin{array}{l}
F(\bar{x}) \in \operatorname{ri}(\operatorname{dom} g-K), \\
G(\bar{x}) \in \operatorname{ri}(-L) .
\end{array}\right.
$$

We point out that a particular version of the following result with $f=0, F$ and $G$ are finite-valued, $K=L=\mathbb{R}_{+}^{n}$, and $g$ is assumed to be $K$-increasing instead of satisfying (7), is proved in [4].

Theorem 35. Under (48), the following are equivalent:

a) $x \in X, G(x) \in-L \Longrightarrow f(x)+g(F(x)) \geq 0$.

b) There exist $\bar{y}, \bar{z}, \bar{s} \in \mathbb{E}_{1}, \bar{u} \in-K^{\circ}, \bar{v} \in-L^{\circ}$ such that

$$
g^{*}(\bar{u})+\langle\bar{u}, F\rangle^{*}(\bar{y})+\langle\bar{v}, G\rangle^{*}(\bar{z})+\sigma_{X}(\bar{s})+f^{*}(-\bar{y}-\bar{z}-\bar{s}) \leq 0 .
$$

Proof. Consider $\mathbb{E}=\mathbb{E}_{2} \times \mathbb{E}_{2}$ equipped with the inner product $\left\langle\left(x_{1}, y_{1}\right),\left(x_{2}, y_{2}\right)\right\rangle:=$ $\left\langle x_{1}, x_{2}\right\rangle+\left\langle y_{1}, y_{2}\right\rangle$ and define $\tilde{K}:=K \times L$ and $\tilde{f}:=f+\delta_{X}$. Let $\tilde{F}: x \in \mathbb{E}_{1} \mapsto$ $(F(x), G(x)) \in \mathbb{E}$ with $\operatorname{dom} \tilde{F}=\operatorname{dom} F \cap \operatorname{dom} G$, and $\tilde{g}:(u, v) \in \mathbb{E} \mapsto g(u)+\delta_{-L}(v)$ with $\operatorname{dom} \tilde{g}=\operatorname{dom} g \times(-L)$ and $\tilde{g}^{*}:(u, v) \mapsto g^{*}(u)+\delta_{-L^{\circ}}(v)$. We then see that 
$\tilde{F}$ is $\tilde{K}$-convex and since $g \in \Gamma\left(\mathbb{E}_{2}\right)$ satisfies (7) and $\delta_{-L}$ is $L$-increasing, $\tilde{g} \in \Gamma(\mathbb{E})$ satisfies (7). In addition, we observe that (48) is equivalent to (17).

a) $\Rightarrow$ b): We note that

$0 \leq \inf _{\substack{x \in X \\ G(x) \in-L}} f(x)+g(F(x))=\inf _{\mathbb{E}_{1}} f+\delta_{X}+g \circ F+\delta_{-L} \circ G=\inf _{\mathbb{E}_{1}} \tilde{f}+\tilde{g} \circ \tilde{F}=-(\tilde{f}+\tilde{g} \circ \tilde{F})^{*}(0)$,

and we conclude that $(\tilde{f}+\tilde{g} \circ \tilde{F})^{*}(0) \leq 0$. We now apply Corollary 16 a) with $\tilde{K}$, $\tilde{f}, \tilde{g}$ and $\tilde{F}$ to get

$$
\begin{aligned}
(\tilde{f}+\tilde{g} \circ \tilde{F})^{*}(0) & =\min _{\substack{(u, v) \in-K^{\circ} \times-L^{\circ} \\
y \in \mathbb{E}_{1}}}\left(f+\delta_{X}\right)^{*}(y)+g^{*}(u)+\delta_{-L^{\circ}}(v)+(\langle u, F\rangle+\langle v, G\rangle)^{*}(-y) \\
& =\min _{\substack{(u, v) \in-K^{\circ} \times-L^{\circ}\\
}} g^{*}(u)+\min _{y \in \mathbb{E}_{1}}\left\{\left(f+\delta_{X}\right)^{*}(y)+(\langle u, F\rangle+\langle v, G\rangle)^{*}(-y)\right\} \\
& =\min _{\substack{(u, v) \in-K^{\circ} \times-L^{\circ} \\
y, z, s \in \mathbb{E}_{1}}} g^{*}(u)+\langle u, F\rangle^{*}(y)+\langle v, G\rangle^{*}(z)+\sigma_{X}(s)+f^{*}(-y-z-s),
\end{aligned}
$$

where the last identity follows from successively applying Theorem 2 which is possible due to (48). The desired implication then follows from (49) and (50) (and the fact that the minimum is taken).

b) $\Rightarrow$ a): We observe that

$$
\begin{aligned}
0 & \geq \inf _{\substack{(u, v) \in-K^{\circ} \times-L^{\circ} \\
y, z, s \in \mathbb{E}_{1}}} g^{*}(u)+\langle u, F\rangle^{*}(y)+\langle v, G\rangle^{*}(z)+\sigma_{X}(s)+f^{*}(-y-z-s) \\
& =\inf _{(u, v) \in-K^{\circ} \times-L^{\circ}} g^{*}(u)+\left(f^{*} \square \sigma_{X} \square\langle u, F\rangle^{*} \square\langle v, G\rangle^{*}\right)(0) \\
& =\inf _{(u, v) \in-K^{\circ} \times-L^{\circ}} g^{*}(u)+\left(\left(f+\delta_{X}\right)^{*} \square(\langle u, F\rangle+\langle v, G\rangle)^{*}\right)(0) \\
& =\inf _{(u, v) \in-K^{\circ} \times-L^{\circ}} g^{*}(u)+\inf _{y \in \mathbb{E}_{1}}\left\{\left(f+\delta_{X}\right)^{*}(y)+(\langle u, F\rangle+\langle v, G\rangle)^{*}(-y)\right\} \\
& =\inf _{y \in \mathbb{E}_{1}}(\tilde{f})^{*}(y)+\inf _{(u, v) \in-K^{\circ} \times-L^{\circ}}\left\{g^{*}(u)+\delta_{-L^{\circ}}(v)+(\langle u, F\rangle+\langle v, G\rangle)^{*}(-y)\right\} \\
& \geq \inf _{y \in \mathbb{E}_{1}}(\tilde{f})^{*}(y)+(\tilde{g} \circ \tilde{F})^{*}(-y) \\
& \geq-\inf _{x \in \mathbb{E}_{1}} \tilde{f}(x)+\tilde{g}(\tilde{F}(x)) \\
& =-\inf _{x \in X, G(x) \in-L} f(x)+g(F(x)),
\end{aligned}
$$

where the third line repeatedly uses Theorem 2 and (48), the sixth line follows from Theorem 12 a), and the seventh is due to (3). The desired implication now follows from (51).

Remark 36. We have the following observations:

a) The result and the proof of Theorem 35 can be extended to the case where $f$ and the convex convex-composite function are finite sums of functions of the same type, respectively.

b) One can also state Theorem $[35$ as a theorem of the alternative: Either

$\left\{\begin{array}{l}x \in X, G(x) \in-L, \\ f(x)+g(F(x))<0\end{array} \quad\right.$ or $\quad\left\{\begin{array}{l}u \in-K^{\circ}, v \in-L^{\circ}, y, z, s \in \mathbb{E}_{1}, \\ g^{*}(u)+\langle u, F\rangle^{*}(y)+\langle v, G\rangle^{*}(z)+\sigma_{X}(s)+f^{*}(-y-z-s) \leq 0\end{array}\right.$ 
has a solution (but not both).

\section{Final remarks}

In this note we developed a full conjugacy and subdifferential calculus for the important subclass of convex-composite functions that are fully convex. Working in the finite-dimensional setting, we took advantage of the relative topology of convex sets, which promoted infimal convolution as our main tool. Combined with the concept of $K$-convexity this enabled us to prove the central convex-analytic results for convex convex-composite functions under weak assumptions (no lower semicontinuity and weak monotonicity properties) on the functions in play, using a verifiable, point-based Slater-type qualification condition. A myriad of applications underlined the versatility of our findings and the unifying strength of the convexcomposite setting even in the fully convex case. In particular, we were able to prove a new extension for the matrix-fractional function to the complex domain, and obtain new and simple proofs for the conjugate and subdifferential of variational Gram functions, an alternative proof of Lewis' result on spectral maps in the convex setting, as well as a refined version of a Farkas-type result due to Bot et al.

For future research we plan on bringing applying the convex-analytic results established here to numerical methods for solving convex convex-composite optimization problems.

\section{Acknowledgments.}

The first author was partially supported by NSF grant DMS1514559. The second author was partially supported by NSERC discovery grant RGPIN-2017-04035. The third author was partially supported by a grant from Centre de recherches de mathématiques (CRM), Montréal.

\section{References}

[1] A. Beck: First-Order Methods in Optimization. MOS-SIAM Series on Optimization, 25. Society for Industrial and Applied Mathematics (SIAM), Philadelphia, PA; Mathematical Optimization Society, Philadelphia, PA, 2017.

[2] D. Bertsekas: Control of Uncertain Systems with a Set-Membership Description of Uncertainty. MOS-PhD Thesis, Massachusetts Institute of Technology, 1971.

[3] J. M. Borwein: Optimization with respect to Partial Orderings. Ph.D. Thesis, University of Oxford, 1974.

[4] R.I. Boţ, I.B. Hodrea, ANd G. WANkA: Farkas-type results for inequality systems with composed convex functions via conjugate duality. Journal of Mathematical Analysis and Applications 322(1), 2006, pp. 316-328.

[5] R.I. Boț, S.-M. Grad, AND G. WANKa: New constraint qualification and conjugate duality for composed convex optimization problems. Journal of Optimization Theory and Applications 135(2), 2007, pp. 241-255.

[6] R.I. Bot And G. WANKA: The conjugate of the pointwise maximum of two convex functions revisited. Journal of Global Optimization 41(3), 2008, pp. 625-632.

[7] R.I. Boţ, S.-M. Grad, AND G. WANKa: A new constraint qualification for the formula of the subdifferential of composed convex functions in infinite dimensional spaces. Mathematische Nachrichten 281(8), 2008, pp. 1088-1107.

[8] R.I. Boţ, S.-M. GRad, And G. WAnka: Generalized Moreau-Rockafellar results for composed convex functions. Optimization 58(7), 2009, pp. 917-933.

[9] R.S. Burachik, V. Jeyakumar, and Z.-Y. Wu: Necessary and sufficient conditions for stable conjugate duality. Nonlinear Analysis 64(9), 2006, pp. 1998-2006. 
[10] J.V. BuRke: Descent methods for composite nondifferentiable optimization problems. Mathematical Programming 33(3), 1985, pp. 260-279.

[11] J.V. Burke: Second order necessary and sufficient conditions for convex composite NDO. Mathematical Programming 38(3), 1987, pp. 287-302.

[12] J.V. BuRKE: An exact penalization viewpoint of constrained optimization. SIAM Journal on Control and Optimization 29(4), 1991, pp. 968-998.

[13] J.V. BuRke AND A. ENGLE: Strong metric (sub)regularity of KKT mappings for piecewise linear-quadratic convex-composite optimization and the quadratic convergence of Newton's method. Mathematics of Operations Research, to appear.

[14] J. V. Burke ANd M. C. Ferris: A Gauss-Newton method for convex composite optimization. Mathematical Programming 71(2), 1995, pp. 179-194.

[15] J. V. Burke, Y. GaO AND T. Hoheisel: Convex geometry of the generalized matrixfractional function. SIAM Journal on Optimization 28(3), 2018, pp. 2189-2200.

[16] J. V. Burke, Y. GaO, AND T. HoheISEl: Variational properties of matrix functions via the generalized matrix-fractional function. SIAM Journal on Optimization 29(3), 2019, pp. 19581987.

[17] J. V. BuRke AND T. HoheISEL: Epi-convergent smoothing with applications to convex composite functions. SIAM Journal on Optimization 23(3), 2013, pp. 1457-1479.

[18] J. V. Burke and T. Hoheisel: Matrix support functionals for inverse problems, regularization, and learning. SIAM Journal on Optimization 25(2), 2015, pp. 1135-1159.

[19] J. V. Burke and R. Poliquin: Optimality conditions for non-finite valued convex composite functions. Mathematical Programming 57(1), Ser. B, 1992, pp. 103-120.

[20] R. Cibulka, A. Dontchev, And A. Kruger: Strong metric subregularity of mappings in variational analysis and optimization. Journal of Mathematical Analysis and Applications 457(2), 2018, pp. 1247-1282.

[21] F.H. Clarke: Optimization and Nonsmooth Analysis. John Wiley \& Sons, New York 1983.

[22] C. Combari, M. Laghdir, And L. Thibault: Sous-différentiels de fonctions convexes composées. Annales des Sciences Mathématiques du Québec 18(2), 1994, pp. 119-148.

[23] Y. Cui, J.S. Pang, and B. Sen: Composite difference-max programs for modern statistical estimation problems. SIAM Journal on Optimization 28(4), 2018, pp. 3344-3374.

[24] D. Davis D And D. Drusvyatskiy: Stochastic model-based minimization of weakly convex functions. SIAM Journal on Optimization 29(1), 2019, pp. 207-239.

[25] S. DenG: On uniqueness of Lagrange multipliers in composite optimization. Journal of Mathematical Analysis and Applications 201(3), 1996, pp. 689-696.

[26] D. DRusvyatskiy D And A.S. Lewis: Error bounds, quadratic growth, and linear convergence of proximal methods. Mathematics of Operations Research 43(3), 2018, pp. 919-948.

[27] D. DRUSVyatskiY AND C. PAQUetTE: Efficiency of minimizing compositions of convex functions and smooth maps. Mathematical Programming, 2018, https://doi.org/10.1007/s10107-018-1311-3

[28] D. Drusvyatskiy and C. Paquette: Variational analysis of spectral functions simplified. Journal of Convex Analysis 25(1), 2018, , pp. 119-134.

[29] J.C. Duchi ANd F. RuAn: Solving (most) of a set of quadratic equalities: Composite optimization for robust phase retrieval. Information and Inference: A Journal of the IMA 8(3), 2019, pp. 471-529.

[30] J.C. Duchi And F. Ruan: Stochastic methods for composite and weakly convex optimization problems. SIAM Journal on Optimization 28(4), 2018, pp. 3229-3259.

[31] S.P. FitzPatrick And S. Simons: On the pointwise maximum of convex functions. Proceedings of the American Mathematical Society 128(12), 2000, pp. 3553-3561.

[32] J.-B. Hiriart-Urruty: A note on the Legendre-Fenchel transform of convex composite functions. In Nonsmooth Mechanics and Analysis. Eds. P. Alart, O. Maisonneuve, and R. T. Rockafellar, Springer, 2006, pp. 35-46.

[33] J.-B. Hiriart-Urruty and C. Lemaréchal: Fundamentals of Convex Analysis. SpringerVerlag, New York, N.Y., 2001.

[34] N. Gaffke and O. Kraffte: Matrix inequalities in the Löwner ordering. In: B. Korte (Ed.), Modern Applied Mathematics, North-Holland, Amsterdam-New York, 1982.

[35] R. Horn and C. R. Johnson: Matrix analysis (2ed). Cambridge University Press, Cambridge, 2013. 
[36] A. Jalali, M. Fazel, and L. XiaO: Variational Gram functions: convex analysis and optimization. SIAM Journal on Optimization 27(4), 2017, pp. 2634-2661.

[37] H. KaWASAKI: Second-order necessary conditions of the Kuhn-Tucker type under new constraint qualifications. Journal of Optimization Theory and Applications 57(2), 1988, pp. 253264.

[38] J. Kiefer: Optimum experimental designs. Journal of the Royal Statistical Society, Series B, Methodological 21, 1959, pp. 272-319.

[39] A.G. Kusraev and S.S. Kutateladze: Subdifferentials: Theory and Applications. Mathematics and its Applications, 323. Kluwer Academic Publishers Group, Dordrecht, 1995.

[40] A.S. LEWIS: The convex analysis of unitarily invariant matrix functions. Journal of Convex Analysis 2(1-2), 1995, pp. 173-183.

[41] A.S. LEwIS: Convex analysis on the hermitian Matrices SIAM Journal on Optimimization 6(1), 1996, pp. 164-177.

[42] A.S. Lewis And S. J. Wright: A proximal method for composite minimization. Mathematical Programming 158(1-2), Series B, 2016, pp. 501-546.

[43] J. von Neumann: Some matrix-inequalities and metrization of matrix-space. Tomsk. Univ. Rev., Vol 1, pp. 286-300, 1937. Reprinted in Collected Works (Pergamon Press, 1962), iv, 205-219.

[44] T. Pennanen: Graph-convex mappings and K-convex functions. Journal of Convex Analysis 6(2), 1999, pp. 235-266.

[45] M.J.D. Powell: Algorithms for nonlinear constraints that use Lagrangian functions. Mathematical programming 14(2), 1978, pp. 224-248.

[46] M.J.D. POWELl: A fast algorithm for nonlinearly constrained optimization calculations. Lecture Notes in Mathematics, Vol. 630, Springer, Berlin, 1978.

[47] R.T. Rockafellar: Convex Analysis. Princeton Mathematical Series, No. 28. Princeton University Press, Princeton, N.J. 1970.

[48] R.T. RockAfELLAR: Extensions of subgradient calculus with applications to optimization. Nonlinear Analysis: Theory, Methods \& Applications 9(7), 1985, pp. 665-698.

[49] R.T. RockAFelLaR: Second-order optimality conditions in nonlinear programming obtained by way of epi-derivatives. Mathematics of Operations Research 14(3), 1989, pp. 462-484.

[50] R.T. Rockafellar and R.J.-B. Wets: Variational Analysis. Grundlehren der Mathematischen Wissenschaften, Vol. 317, Springer-Verlag, Berlin, 1998.

[51] A. Shapiro And K. Scheinberg:Duality and optimality conditions. In: Handbook of Semidefinite Programming: Theory, Algorithms, and Applications. Eds.: H. Wolkowicz, R. Saigal, L. Vandenberghe. Springer Science+Business Media, Springer, New York, 2003.

[52] S.J. WRIGHT: Local properties of inexact methods for minimizing nonsmooth composite functions. Mathematical programming 37(2), 1987, pp. 232-252.

[53] Y. YuAN: On the superlinear convergence of a trust region algorithm for nonsmooth optimization. Mathematical Programming 31(3), 1986, pp. 269-285.

[54] C. ZĂLINESCU: Duality for vectorial nonconvex optimization by convexification and applications. Analele Ştiinţifice ale Universităţii "Al. I. Cuza" din Iaşi. Secţiunea I a Matematică 29(3), 1983, pp. 15-34.

Department of Mathematics, University of Washington, 7521 - 30th Ave. N.E., SeatTLE, WA 98115, USA

E-mail address: jvburke01@gmail.com

Department of Mathematics and Statistics, McGill University, 805 Sherbrooke St West, Room 1114, Montréal, Québec, Canada H3A 0B9

E-mail address: tim.hoheisel@mcgill.ca

Department of Mathematics and Statistics, McGill University, 805 Sherbrooke St West, Montréal, QuéBec, Canada H3A 0B9

E-mail address: van.q.nguyen@mcgill.ca 